\title{
MicroRNA-29a suppresses the invasion and migration of osteosarcoma cells by regulating the SOCS1/NF-кB signalling pathway through negatively targeting DNMT3B
}

\author{
HAO-LI GONG ${ }^{1}$, YE TAO ${ }^{2}$, XIN-ZHAN MAO ${ }^{1}$, DE-YE SONG $^{1}$, DI YOU ${ }^{1}$ and JIANG-DONG NI ${ }^{1}$ \\ ${ }^{1}$ Department of Orthopedics, The Second Xiangya Hospital, Central South University, Changsha, Hunan 410008; \\ ${ }^{2}$ Department of Radiology, The Third Xiangya Hospital, Central South University, Changsha, Hunan 410013, P.R. China
}

Received October 8, 2018; Accepted May 31, 2019

DOI: $10.3892 /$ ijmm.2019.4287

\begin{abstract}
The present study aimed to investigate the roles of the microRNA-29a/DNA methyltransferase 3B/suppressor of cytokine signalling 1 (miR-29a/DNMT3B/SOCS1) axis in the invasion and the migration of osteosarcoma (OS). The expression levels of miR-29a, DNMT3B and SOCS1 were determined in tissue samples and OS cell lines by reverse transcription-quantitative polymerase chain reaction (PCR). Apoptosis was measured using flow cytometry analysis. Transwell and wound healing assays were conducted to measure the invasion and migration abilities of OS cells, respectively. A dual-luciferase reporter assay was also conducted to determine the interaction between DNMT3B and miR-29a, while methylation-specific PCR was used to detect the methylation of SOCS1. Western blotting was performed to determine the protein levels of DNMT3B and SOCS1, as well as the levels of proteins associated with epithelial-mesenchymal transition (EMT), apoptosis and the nuclear factor (NF)- $\mathrm{kB}$ signalling pathway. The results demonstrated that miR-29a and SOCS1 were downregulated, and DNMT3B was upregulated in both OS tissues and cell lines. The expression of miR-29a and SOCS1 was found to be associated with advanced clinical stage and distant metastasis. In addition, the dual-luciferase reporter assay revealed that DNMT3B was a direct target of miR-29a. Overexpression using miR-29a mimics decreased DNMT3B expression and the methylation level of SOCS1, which resulted in the upregulation of SOCS1 in U2OS and MG-63 cells, while miR-29a inhibition led to the opposite results. Transfection with miR-29a mimics also promoted the apoptosis, and inhibited the invasion, migration and EMT process of OS cells, while it
\end{abstract}

Correspondence to: Dr Jiang-Dong $\mathrm{Ni}$, Department of Orthopedics, The Second Xiangya Hospital, Central South University, 139 Middle Renmin Road, Changsha, Hunan 410008, P.R. China

E-mail: nijiangdong001@csu.edu.cn

Key words: osteosarcoma, metastasis, microRNA-29a, DNA methyltransferase $3 \mathrm{~B}$, suppressor of cytokine signalling 1 , nuclear factor- $\kappa \mathrm{B}$ signalling pathway markedly reduced the nuclear translocation of p65 and IкB- $\alpha$ degradation. Treatment with 5-aza-2'-deoxycytidine worked together with miR-29a mimics to synergistically enhance the aforementioned effects. By contrast, the effects induced by miR-29a were partly reversed upon co-transfection with SOCS1 siRNA. In conclusion, miR-29a promoted the apoptosis, and inhibited the invasion, migration and EMT process of OS cells via inhibition of the SOCS1/NF- $\kappa$ B signalling pathway by directly targeting DNMT3B.

\section{Introduction}

Osteosarcoma (OS), the most frequent malignant bone tumour, often occurs in children, accounting for $2.4 \%$ of all malignancies and $20 \%$ of all primary bone tumours in paediatric patients $(1,2)$. Despite the rapid advances in treatment and diagnosis, the survival of OS patients remains poor, and its recurrence rate is as high as $30-40 \%$ due to its highly aggressive and metastatic properties $(3,4)$. The current standard chemotherapy methods only provide $65-70 \%$ long-term disease-free survival for OS patients without metastasis, and $70 \%$ OS patients with recurrence die within 5 years $(5,6)$. Thus, it is of high urgency to uncover the mechanisms of OS tumourigenesis, particularly its invasion and migration.

MicroRNAs (miRNAs) are small endogenous RNAs that are involved in various steps of tumourigenesis, including in cell proliferation, apoptosis, migration and invasion $(7,8)$. miR-29a, which is considered as a tumour suppressor, serves an important role in the development of several types of cancer, such as lung (9), prostate (10) and gastric cancer (11). A previous study has also reported that miR-29a is downregulated in OS (12); however, the involvement and mechanisms for miR-29a in the development of OS have rarely been reported (12). The hypermethylation of certain tumour suppressor gene promoters plays a crucial role in cancer progression, and the interaction between methylation of anti-oncogenes and miRNAs has been demonstrated in several types of cancer $(13,14)$. Recently, it was reported that overexpression of DNA methyltransferase 3B (DNMT3B) was correlated to the downregulation of miR-29a in juvenile myelomonocytic leukaemia (15). However, to the best of our knowledge, no study has investigated the association between DNMT3B and miR-29a in OS. 
The suppressor of cytokine signalling (SOCS) family consists of eight members, namely SOCS1-7, as well as the cytokine-inducible SH2-containing protein (16). SOCS1 can act as both tumour suppressor and oncogene in cancer development (17), and the methylation of SOCS1 promoter regions by methyltransferases is reportedly involved in the tumourigenesis of several cancer types. The methylation of SOCS1 promotes the growth and proliferation of acute myeloid leukaemia cells through the JAK2/STAT signalling pathway (18). In addition, SOCS1 has been demonstrated to suppress the metastasis of melanoma cells (19) and human prostate cancer cells (20). However, SOCS1 can also act as an oncogene, and silencing SOCS1 in monocytes enhanced the tumour-killing activity of macrophages (21). A previous study demonstrated that SOCS1 was involved in the development of OS (22). However, deeper insights on the function of SOCS1 in OS are still needed, and no study has focussed on the association between the miR-29a/DNMT3B axis and SOCS1, or its influence on the apoptosis, invasion and migration of OS cells.

The SOCS1/nuclear factor (NF)- $\kappa B$ pathway serves a vital role in inflammatory processes $(23,24)$. SOCS1 regulates the epithelial-mesenchymal transition (EMT) and bone metastasis of prostate cancer via the NF- $\kappa \mathrm{B}$ signalling pathway (25). SOCS1, the key factor in our research, is an upstream protein of NF- $\mathrm{KB}$ (26), while several studies have reported that the NF- $\mathrm{KB}$ signalling pathway is associated with tumour invasion and migration. It was also demonstrated that $\mathrm{NF}-\mathrm{\kappa B}$ promoted EMT, migration and invasion in pancreatic carcinoma cells (27). Furthermore, a previous review demonstrated that activation of the TNF- $\alpha / N F-\kappa B$ pathway may contribute to tumor development and promote cell migration and invasion by activating Snail and epithelial-mesenchymal transformation (28). Therefore, we hypothesized that miR-29a may modulate tumourigenesis in OS by regulating the SOCS1/NF- $\kappa \mathrm{B}$ signalling pathway by directly targeting DNMT3B.

In the present study, it was observed that miR-29a promoted apoptosis, and inhibited invasion, migration and EMT in OS cells via the SOCS1/NF- $\mathrm{kB}$ signalling pathway by targeting DNMT3B. To the best of our knowledge, this is the first study examining the association between miR-29a, DNMT3B and SOCS1 in OS tumourigenesis. The current study may provide a better understanding of the miR-29a/DNMT3B/SOCS1 axis in the development of OS and provide novel therapeutic targets for OS treatment.

\section{Materials and methods}

Tissue samples. A total of 30 paired OS tissues and adjacent non-tumour tissue samples were used in the present study, which were obtained from patients who underwent radical resection at the Second Xiangya Hospital, Central South University (Changsha, China). Samples were frozen immediately after surgical resection and stored in liquid nitrogen until required for further assay. Histological analysis was conducted to confirm the pathology of all tissues (29). Written informed consent was obtained from all patients, and the study was approved by the Ethics Committee of the Second Xiangya Hospital, Central South University. The clinicopathological features of all OS patients are listed in Table I.
Cell culture and transfection. The OS cell lines U2OS, MG-63 and Saos-2, as well as the normal hFOB 1.19 osteoblast cells, were all purchased from ATCC (Manassas, VA, USA). Briefly, cells were cultured in Dulbecco's modified Eagle's medium (DMEM; Thermo Fisher Scientific, Inc., Waltham, MA, USA) containing $10 \%$ foetal bovine serum (FBS; Gibco; Thermo Fisher Scientific, Inc.) and $100 \mu \mathrm{g} / \mathrm{ml}$ penicillin-streptomycin (Sigma-Aldrich; Merck KGaA, Darmstadt, Germany) at $37^{\circ} \mathrm{C}$ and $5 \% \mathrm{CO}_{2}$. When the OS cells reached $70-80 \%$ confluence, they were transfected with miR-29a mimics, miR-29a inhibitor, negative control (NC) mimics, NC inhibitor (all $10 \mathrm{nM}$; GeneChem Corp., Shanghai, China), SOCS1 siRNA (siSOCS1) or NC siRNA (siNC) for $48 \mathrm{~h}$ using the Lipo6000 transfection reagent (Beyotime Institute of Biotechnology, Haimen, China) according to the manufacturer's protocol. After $48 \mathrm{~h}$, the transfection rate was determined using RT-qPCR. To inhibit the level of methylation, 5-aza-2'-deoxycytidine (5-Aza; $10 \mu \mathrm{M}$; Sigma-Aldrich; Merck KGaA, Darmstadt, Germany) was added to the cells. Briefly, U2OS or MG63 cells were treated with $10 \mu \mathrm{M}$ 5-Aza after cells reached 70-80\% confluence or cells were treated with $10 \mu \mathrm{M} 5$-Aza after transfection of miR-29a mimics for $24 \mathrm{~h}$. Cells not treated with 5-Aza were used as controls. The cells were further cultured for $24 \mathrm{~h}$ for further experiments.

293T cells (American Type Culture Collection) were also cultured in DMEM (Thermo Fisher Scientific, Inc.) containing $10 \%$ FBS (Gibco; Thermo Fisher Scientific, Inc.) and $100 \mu \mathrm{g} / \mathrm{ml}$ penicillin-streptomycin at $37^{\circ} \mathrm{C}$ and $5 \% \mathrm{CO}_{2}$.

Dual-luciferase reporter assay. The predicted binding region of DNMT3B and miR-29a was obtained using Targetscan 7.2 (http://www.targetscan.org). To confirm whether DNMT3B was a direct target of miR-29a, a dual-luciferase reporter assay was conducted. Briefly, the DNMT3B 3'-untranslated region with the predicted miR-29a binding site (WT) or mutant binding site (MUT) was amplified, and then sub-cloned into the pGL4.10 luciferase reporter vector. 293T cells were then co-transfected with the miR-29a mimics or NC mimics using the Lipo6000 reagent (Beyotime Institute of Biotechnology) according to the manufacturer's protocol. After $48 \mathrm{~h}$ of transfection, a luciferase reporter assay was performed using a Bright-Glo ${ }^{\mathrm{TM}}$ Luciferase Assay System (Promega Corporation, Madison, WI, USA). The luciferase activity was normalised to the value of the Renilla luciferase activity.

Apoptosis assay. To study the effect of miR-29a on apoptosis, cells were stained with an Annexin V/propidium iodide (PI) double staining kit (BD Biosciences, Franklin Lakes, NJ, USA) according to the manufacturer's protocol. Briefly, the cells were seeded at density of $3 \times 10^{5}$ in 24 -well plates, and were collected after $48 \mathrm{~h}$ of transfection, washed twice with cold PBS and resuspended in $1 \mathrm{X}$ binding buffer. Cells were stained with $5 \mu \mathrm{l}$ Annexin V-FITC for $15 \mathrm{~min}$ and then with $5 \mu \mathrm{PI}$ for $10 \mathrm{~min}$ in the dark at room temperature. Apoptosis was measured by flow cytometry (BD Biosciences).

Wound healing assay. Cell migration was determined by performing a scratch-wound healing assay. Briefly, cells at density of $1 \times 10^{6} /$ well were seeded into 6-well plates for $24 \mathrm{~h}$. Cell layers were scratched using a $100-\mu 1$ pipette tip to form 
Table I. Association of miR-29a, DNMT3B and SOCS1 expression with the clinicopathological characteristics of osteosarcoma patients.

\begin{tabular}{|c|c|c|c|c|c|c|c|c|c|c|}
\hline \multirow[b]{2}{*}{ Clinical parameters } & \multirow[b]{2}{*}{ Cases (n) } & \multicolumn{2}{|c|}{$\begin{array}{l}\text { miR-29a } \\
\text { expression }\end{array}$} & \multirow[b]{2}{*}{ P-value } & \multicolumn{2}{|c|}{$\begin{array}{l}\text { DNMT3B } \\
\text { expression }\end{array}$} & \multirow[b]{2}{*}{ P-value } & \multicolumn{2}{|c|}{$\begin{array}{c}\text { SOCS1 } \\
\text { expression }\end{array}$} & \multirow[b]{2}{*}{ P-value } \\
\hline & & High & Low & & High & Low & & High & Low & \\
\hline \multicolumn{11}{|l|}{ Age (years) } \\
\hline$<18$ years & 20 & 11 & 9 & 0.6999 & 8 & 12 & 0.2451 & 9 & 11 & 0.6999 \\
\hline$\geq 18$ years & 10 & 4 & 6 & & 7 & 3 & & 6 & 4 & \\
\hline \multicolumn{11}{|l|}{ Sex } \\
\hline Male & 17 & 6 & 11 & 0.1394 & 10 & 7 & 0.4621 & 12 & 5 & 0.0253 \\
\hline Female & 13 & 9 & 4 & & 5 & 8 & & 3 & 10 & \\
\hline \multicolumn{11}{|l|}{ Tumor size $(\mathrm{cm})$} \\
\hline$<5 \mathrm{~cm}$ & 12 & 7 & 5 & 0.7104 & 4 & 8 & 0.2635 & 3 & 9 & 0.0604 \\
\hline$\geq 5 \mathrm{~cm}$ & 18 & 8 & 10 & & 11 & 7 & & 12 & 6 & \\
\hline \multicolumn{11}{|l|}{ TNM stage } \\
\hline I & 14 & 12 & 2 & 0.0007 & 4 & 10 & 0.0656 & 11 & 3 & 0.0092 \\
\hline II+III & 16 & 3 & 13 & & 11 & 5 & & 4 & 12 & \\
\hline \multicolumn{11}{|l|}{ Distant metastasis } \\
\hline Yes & 16 & 4 & 12 & 0.0092 & 10 & 6 & 0.2723 & 14 & 2 & $<0.0001$ \\
\hline No & 14 & 11 & 3 & & 5 & 9 & & 1 & 13 & \\
\hline
\end{tabular}

miR-29a, microRNA-29a; SOCS1, suppressor of cytokine signalling 1; DNMT3B, DNA methyltransferase 3B.

a wound-like gap. The cells were then maintained in DMEM with $10 \%$ FBS, and images were captured at 0 and $24 \mathrm{~h}$. ImageJ software (National Institutes of Health, Bethesda, MD, USA) was used to analyse the wound width. The migration distance of cells was measured according to the following formula: Migration rate $(\%)=\left(\mathrm{W}_{0 \mathrm{~h}}-\mathrm{W}_{24 \mathrm{~h}}\right) / \mathrm{W}_{0 \mathrm{~h}} \times 100 \%$.

Transwell assay. A Transwell assay was used to determine the cell invasion ability. Briefly, $2 \times 10^{5}$ cells were plated in the top chamber of 24-well Transwell inserts with a Matrigel-coated membrane (BD Biosciences) in serum-free medium. As a chemoattractant, DMEM with $20 \%$ FBS was added to the bottom compartment. Cells were cultured for $24 \mathrm{~h}$ at $37^{\circ} \mathrm{C}$, and the non-invading cells on the upper surface were removed with a cotton swab. The invaded cells on the lower surface were fixed with $4 \%$ paraformaldehyde and stained with $0.1 \%$ crystal violet. Invading cells were finally counted under an inverted microscope (Zeiss, Oberkochen, Germany).

RNA extraction and reverse transcription-quantitative polymerase chain reaction $(R T-q P C R)$ assay. The expression levels of miR-29a, DNMT3B and SOCS1 were measured using RT-qPCR. Briefly, the extraction of total RNA was conducted using the TRIzol reagent (Tiangen Biotech, Beijing, China). For extraction of miRNA, the mirVana miRNA isolation kit (Ambion; Thermo Fisher Scientific, Inc.) was used. The RNA concentration was determined using a NanoDrop ND-1000 spectrophotometer (NanoDrop Technologies; Thermo Fisher Scientific, Inc.). Subsequently, a Prime-Script ${ }^{\mathrm{TM}}$ One Step RT-qPCR kit (Takara Biotechnology Co., Ltd., Dalian, China) and a TaqMan MicroRNA Reverse Transcription kit (Applied Biosystems Life Technologies) were used to convert RNA to cDNA for mRNA and miRNA, respectively. Subsequently, qPCR analysis was performed in an Applied Biosystems 7500 Real Time PCR system (Applied Biosystems; Thermo Fisher Scientific, Inc.) using SYBR Green PCR Master Mix (Solarbio Science \& Technology Co., Ltd., Beijing, China) using the following conditions: Initial activation step at $95^{\circ} \mathrm{C}$ for $5 \mathrm{sec}, 35$ cycles of denaturation at $94^{\circ} \mathrm{C}$ for $15 \mathrm{sec}$, annealing at $55^{\circ} \mathrm{C}$ for $25 \mathrm{sec}$, and extension at $70^{\circ} \mathrm{C} 30 \mathrm{sec}$. The primers used in qPCR were as follows: miR-29a forward, 5'-CGCGGATCCTGGATTTAGTAAGAT TTGGGC-3', and reverse, 5'-CCGGAATTCACATGCAAT TCAGGTCAGTG-3'; DNMT3B forward, 5'-GCCCATTCG ATCTGGTGATT-3', and reverse, 5'-GGCGGTAGAACT CAAAGAAGAG-3'; SOCS1 forward, 5'-TGGTTGTAGCAG CTTGTGTCTGG-3', and reverse, 5'-CCTGGTTTGTGC AAAGATACTGGG-3'; U6 snRNA forward, 5'-ATTGGA ACGATACAGAGAAGATT-3', and reverse 5'-GGAACG CTTCACGAATTTG-3'; GAPDH forward, 5'-CCACAGTCC ATGCCATCAC-3', and reverse 5'-GCTTCACCACCTTCT TGATG-3'. GAPDH and U6 small nuclear RNA (U6 snRNA) were used as internal references for mRNA and miRNA, respectively. The relative expression level was calculated by the $2^{-\Delta \Delta \mathrm{Cq}}$ method (30).

Methylation-specific PCR (MSP) assay. For analysis of the methylation level of SOCS1, the MSP method was used. Briefly, DNA was isolated from the cell lines using a QIAamp Fast DNA Tissue kit (Qiagen, Valencia, CA, USA). Subsequent to transforming unmethylated cytosine residues to uracil using an EpiTect MSP kit (Qiagen), the bisulphite-treated DNA was 
amplified using the following MSP primers: SOCS1 (methylated samples) forward, 5'-TTCGCGTGTATTTTTAGGTCG GTC-3', and reverse, 5'-CGACACAACTCCTACAACGAC CG-3'; SOCS1 (unmethylated samples) forward, 5'-TTATGA GTATTTGTGTGTATTTTTAGGTTGGTT-3', and reverse, 5'-CACTAACAACACAACTCCTACAACAACCA-3'. The PCR reaction and quantification methods were conducted as aforementioned.

Western blot assay. Western blotting was used to test the protein levels of DNMT3B and SOCS1, as well as the levels of proteins associated with EMT, apoptosis and the NF- $\mathrm{BB}$ signalling pathway. Briefly, total proteins were extracted from the cells or tissues using RIPA lysis buffer (Cell Signaling Technology, Inc., Danvers, MA, USA) containing a protease inhibitor cocktail (Roche, Mannheim, Germany). Nuclear proteins were extracted using a nucleoprotein kit (Merck KGaA, West Point, PA, USA) according to the manufacturer's protocol. Next, the protein concentration was determined using a BCA Protein Assay kit (Pierce; Thermo Fisher Scientific, Inc.). Samples were then separated by $10 \%$ SDS-PAGE, transferred to PVDF membranes, blocked by $5 \%$ non-fat milk at room temperature for $1 \mathrm{~h}$ and incubated at $4^{\circ} \mathrm{C}$ overnight with a corresponding primary antibody: DNMT3B (ab79822; 1:1,000), SOCS1 (ab9870; 1:2,000), fibronectin (ab2413; 1:1,000), vimentin (ab8978; 1:1,000), $\mathrm{N}$-cadherin (ab18203; 1:500), E-cadherin (ab15148; 1:500), matrix metalloproteinase (MMP)-2 (ab37150; 1:2,000), MMP-9 (ab73734; 1:2,000), p65 (ab16502; 1:1,000), IкB $\alpha$ (ab32518; 1:1,000), p-IкB- $\alpha$ (ab133462; 1:10,000), cleaved caspase-3 (ab13847; 1:500), pro-caspase-3 (ab32499; 1:10,000), cleaved poly(ADP-ribose) polymerase (PARP; ab4830; 1:1,000), PARP (ab74290; 1:1,000), B-cell lymphoma-2 (Bcl-2; ab196495; 1:500), Bcl-2-associated X protein (Bax; ab53154; 1:500) and GAPDH (ab8245; 1:2,000). Samples were then incubated with horseradish peroxidase-conjugated anti-rabbit immunoglobulin G secondary antibody (ab6721; 1:1,000) at room temperature for $45 \mathrm{~min}$. All primary and secondary antibodies were obtained from Abcam (Cambridge, UK). The signal was developed using an ECL system (Beyotime Institute of Biotechnology) according to the manufacturer's protocol. The protein levels were quantified using Quantity One software (Bio-Rad Laboratories, Inc., Hercules, CA, USA), and GAPDH was used as an internal reference.

Statistical analysis. All experiments were repeated at least three times, and one representative result is presented. Data were analysed with Prism software, version 6.0 (GraphPad Software, San Diego, CA, USA). The data are expressed as the mean \pm standard deviation. Comparison between two groups in Fig. 1 was performed using paired Student's $t$ test, while comparison between two groups in other figures using unpaired Student's $t$ test. Comparison among three or more groups was conducted using one-way analysis of variance, followed by Tukey's post-hoc test. The correlations between miR-29a/DNMT3B/SOCS1 expression level and the clinicopathological characteristics of patients with OS were assessed by the $\chi^{2}$ test. A P-value of $<0.05$ was considered to denote that the difference was statistically significant.

\section{Results}

miR-29a and SOCS1 are downregulated, and DNMT3B is upregulated in OS tissues. The levels of miR-29a, DNMT3B and SOCS1 in OS and adjacent normal tissues were determined by RT-qPCR and western blot analysis. The results demonstrated that, in OS tissues, miR-29a and SOCS1 were significantly downregulated, and DNMT3B was significantly upregulated compared with the adjacent non-tumour tissues (Fig. 1A). The protein level of SOCS1 was also markedly decreased in tumour tissues, while DNMT3B was significantly increased (Fig. 1B and C). As shown in Table I, miR-29a and SOCS1 expression were found to be associated with an advanced clinical stage $(\mathrm{P}<0.05)$ and distant metastasis $(\mathrm{P}<0.05)$. All these results suggested that miR-29a and SOCS1 may be tumour biomarkers of OS progression.

miR-29a and SOCS1 are downregulated, and DNMT3B is upregulated in OS cells. To further investigate the mechanisms of the miR-29a/DNMT3B/SOCS1 axis in OS, the levels of miR-29a, DNMT3B and SOCS1 were determined in OS cells. As shown in Fig. 2A, in the three OS cell lines investigated, miR-29a and SOCS1 were significantly downregulated, while DNMT3B was significantly upregulated compared with the normal hFOB 1.19 cells, which is consistent with the results in tissue samples. In addition, the MSP assay revealed that the methylation level of SOCS1 was significantly increased in OS cells in comparison with that in hFOB 1.19 cells (Fig. 2B). Furthermore, protein level changes of DNMT3B and SOCS1 were similar to the alterations at the mRNA level: The expression of SOCS1 was significantly inhibited, while that of DNMT3B was significantly induced in OS cells (Fig. 2C and D). The migration assay revealed that migration distances in all OS cell lines were significantly higher compared with those in hFOB 1.19 cells, suggesting that $\mathrm{OS}$ cells exhibit strong migration ability. Among the three OS cells, U2OS and MG-63 cells exhibited stronger migration abilities in comparison with the Saos-2 cells (Fig. 2E and F). Considering their migration ability and expression of miR-29a, DNMT3B and SOCS1, the U2OS and MG-63 cell lines were selected for use in subsequent experiments.

miR-29a overexpression downregulates the methylation of SOCSI and upregulates SOCS1 expression through negatively targeting DNMT3B. The miR-29a mimics and the methyltransferase inhibitor 5-Aza were used to treat U2OS and MG-63 cells. The results demonstrated that miR-29a was significantly upregulated in the two cell lines following transfection with the miR-29a mimics (Fig. 3A), while co-treatment with 5-Aza did not markedly affect miR-29a expression. Furthermore, the overexpression of miR-29a, as well as the treatment with 5-Aza, significantly downregulated DNMT3B and upregulated SOCS1 levels, while the combination treatment with miR-29a mimics and 5-Aza further enhanced these effects (Fig. 3B and C). By contrast, when cells were transfected with miR-29a inhibitor, the opposite results were observed. Upon inhibition of miR-29a, the expression levels of miR-29a and SOCS1 were significantly downregulated, while DNMT3B was markedly elevated (Fig. 3D). In the methylation analysis of SOCS1, it was observed that the methylation level was significantly inhibited when cells were transfected with miR-29a mimics or treated with 5-Aza, and miR-29a mimics synergised 
A

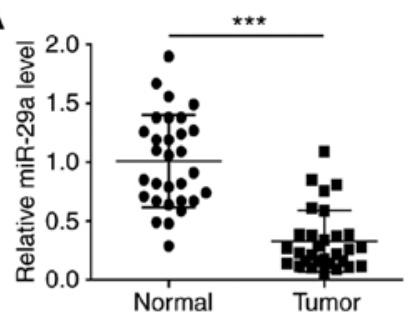

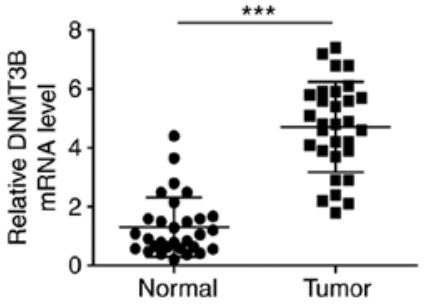

C

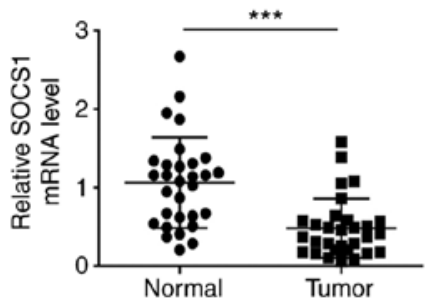

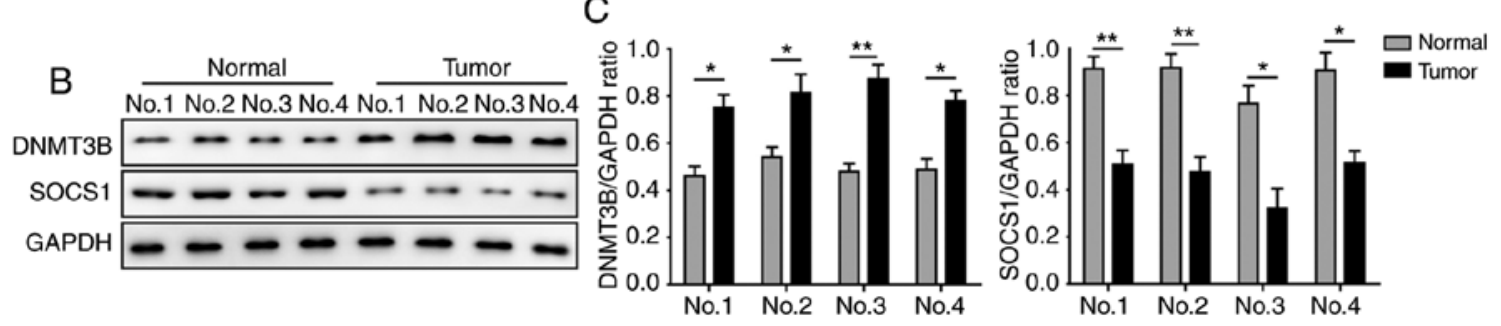

Figure 1. miR-29a and SOCS1 are downregulated and DNMT3B is upregulated in OS tissues. (A) Expression of miR-29a, DNMT3B and SOCS1 in OS and normal tissues, assessed by reverse transcription-quantitative polymerase chain reaction. (B) Protein levels of DNMT3B and SOCS1 in OS and normal tissues, assessed by western blotting. (C) Quantified results of DNMT3B and SOCS1 protein levels in tissues. Data are expressed as the mean \pm standard deviation. Comparison between two groups was performed using paired Student's t-test. ${ }^{*} \mathrm{P}<0.05,{ }^{* *} \mathrm{P}<0.01$ and ${ }^{* * * *} \mathrm{P}<0.001$. miR-29a, microRNA-29a; SOCS1, suppressor of cytokine signalling 1; DNMT3B, DNA methyltransferase 3B; OS, osteosarcoma.

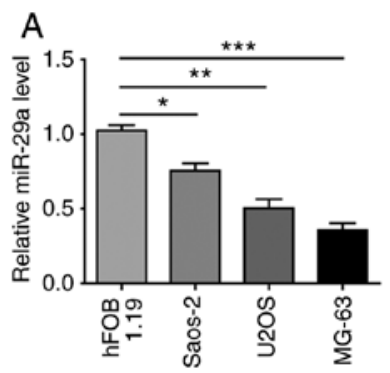

B
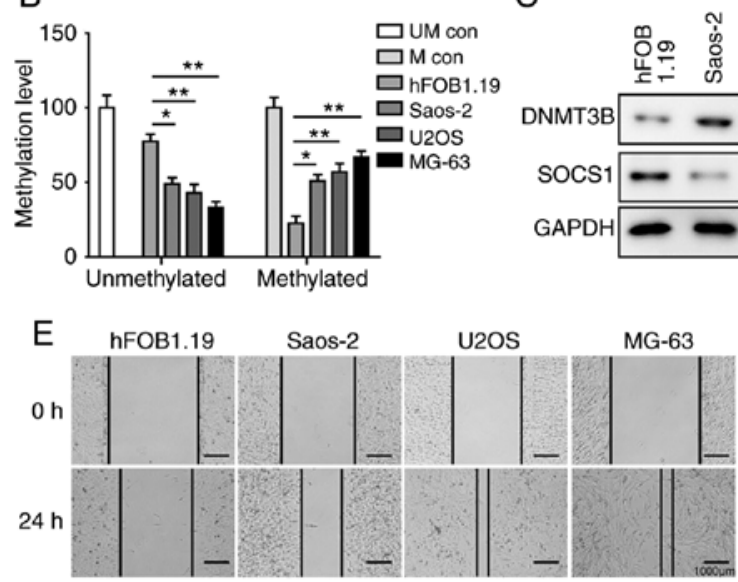

C
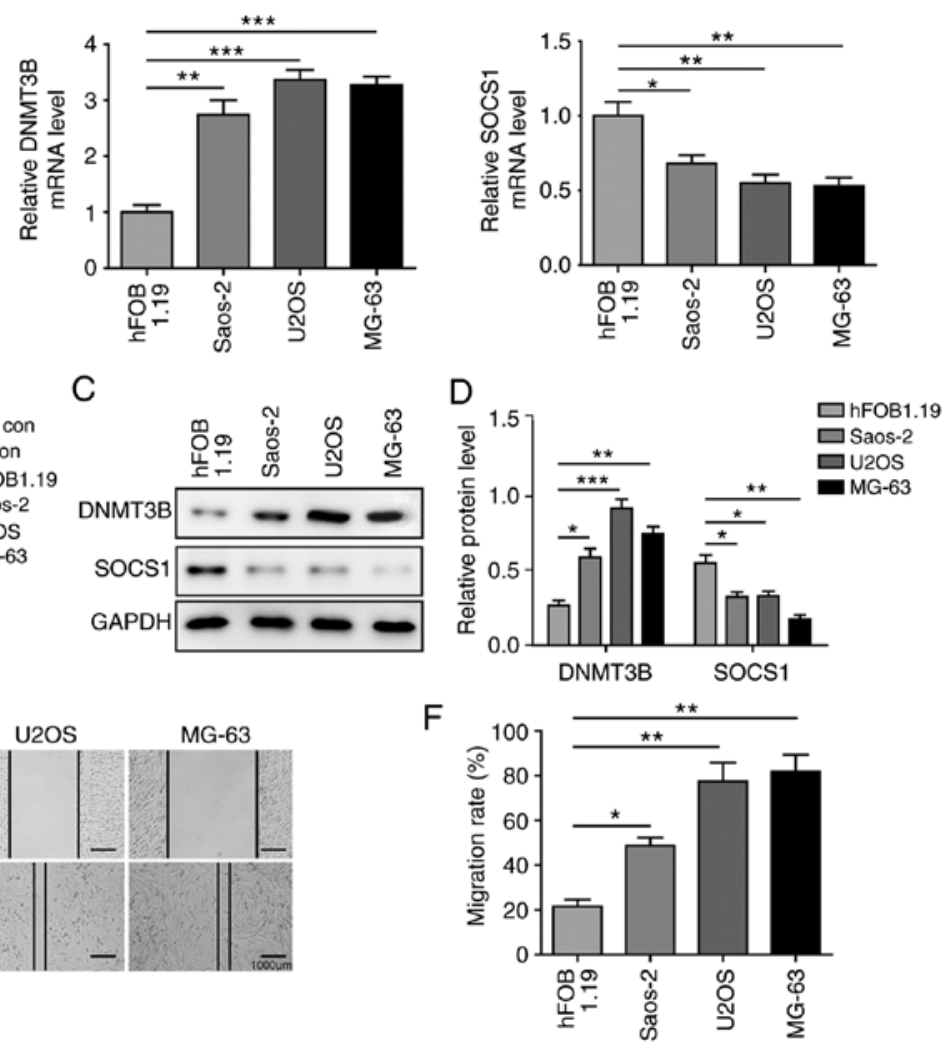

Figure 2. miR-29a and SOCS1 are downregulated, and DNMT3B is upregulated in OS cells. (A) Expression levels of miR-29a, DNMT3B and SOCS1 in different OS cell lines and normal hFOB1.19 cells, assessed by reverse transcription-quantitative PCR. (B) Methylation level of SOCS1 in different OS cell lines and normal hFOB1.19 cells, examined by methylation-specific PCR. (C) Western blots and (D) quantified protein levels of DNMT3B and SOCS1 in three OS cell lines and normal hFOB1.19 cells. (E) Representative wound healing assay images (magnification, $\mathrm{x} 40)$ and (F) migration distance of different OS cell lines. Data are expressed as the mean \pm standard deviation. Comparison among three or more groups was conducted using one-way analysis of variance followed by Tukey's post hoc test. ${ }^{*} \mathrm{P}<0.05,{ }^{* *} \mathrm{P}<0.01$ and ${ }^{* * *} \mathrm{P}<0.001$. miR-29a, microRNA-29a; SOCS1, suppressor of cytokine signalling 1 ; DNMT3B, DNA methyltransferase 3B; OS, osteosarcoma; PCR, polymerase chain reaction; UM con, unmethylated control; M con, methylated control.

with 5-Aza to significantly exacerbate this effect (Fig. 3E). Alteration of the protein levels of DNMT3B and SOCS1 was consistent with the mRNA results (Fig. 4A-D).
To further investigate the aforementioned changes, a dual-luciferase reporter assay was conducted to determine whether DNMT3B was a direct target of miR-29a. The binding 

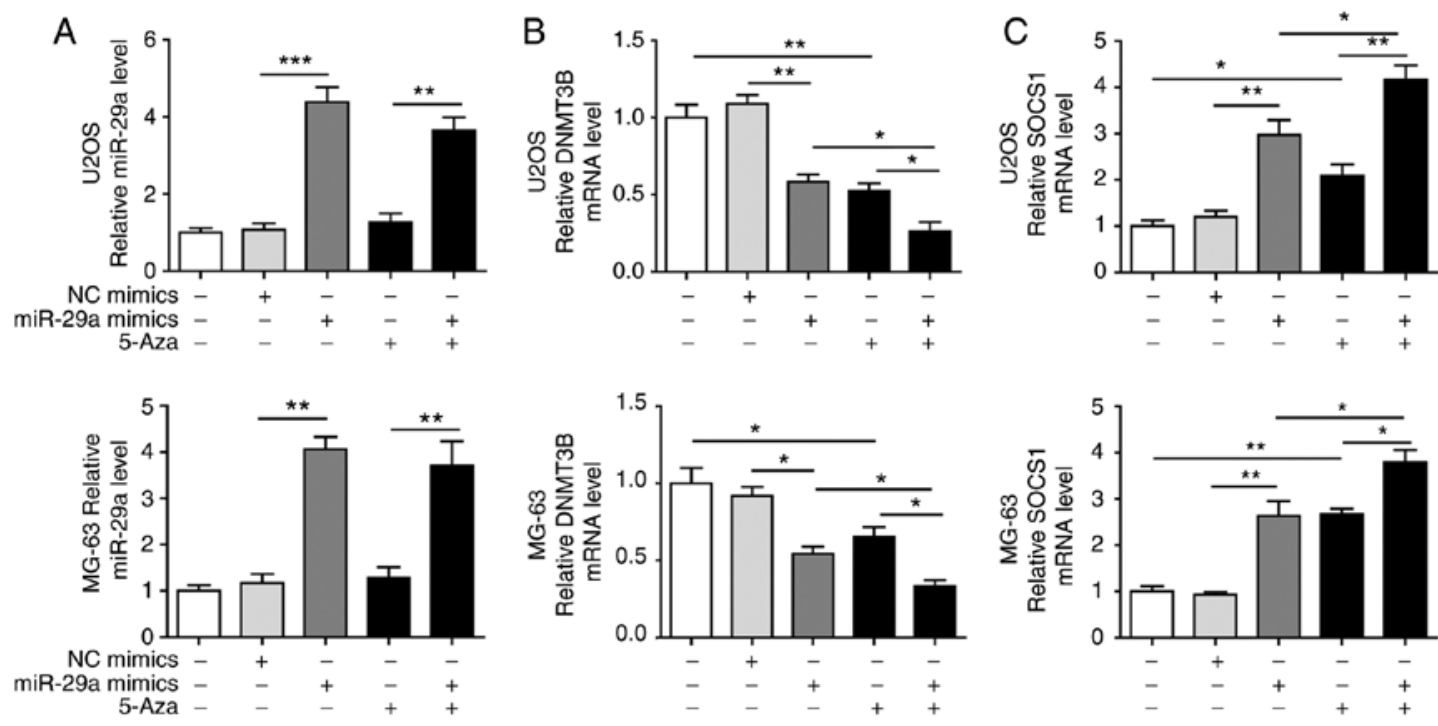

$\mathrm{D}$

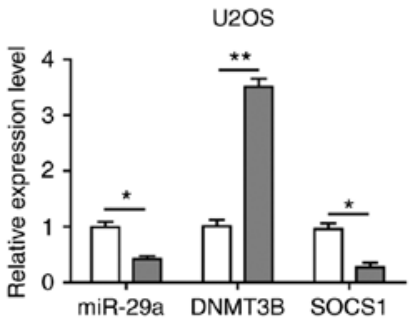

$\mathrm{E}$

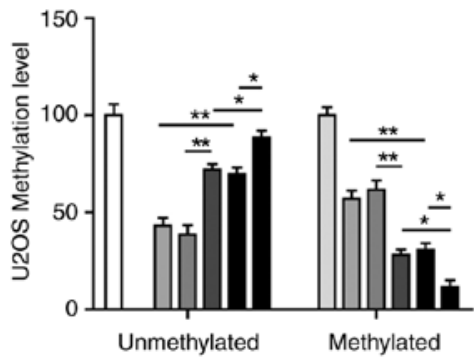

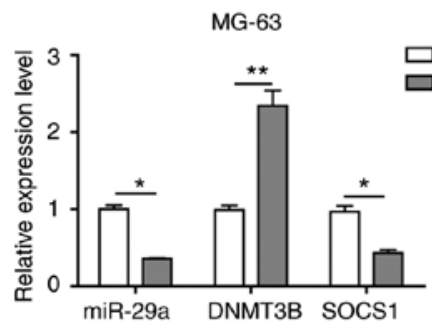

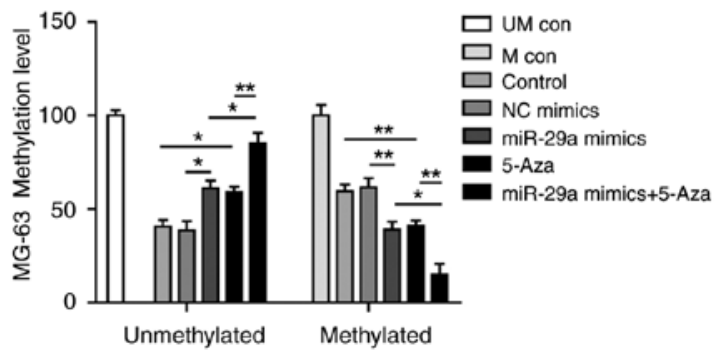

Figure 3. miR-29a downregulates the methylation of SOCS1 and upregulates SOCS1 expression in U2OS and MG-63 cells. (A) miR-29a, (B) DNMT3B and (C) SOCS1 expression levels in cells transfected with miR-29a mimics and/or treated with 5-Aza. (D) Expression of miR-29a, DNMT3B and SOCS1 in cells transfected with miR-29a inhibitor or NC, as measured by reverse transcription-quantitative polymerase chain reaction. (E) Methylation level of SOCS1 in cells transfected with miR-29a mimics and/or treated with 5-Aza. Comparison between two groups was performed using unpaired Student's t-test, while comparison among three or more groups was conducted using one-way analysis of variance followed by Tukey's post hoc test. Data are expressed as the mean \pm standard deviation. ${ }^{*} \mathrm{P}<0.05,{ }^{* *} \mathrm{P}<0.01$ and ${ }^{* * *} \mathrm{P}<0.001$. miR-29a, microRNA-29a; SOCS1, suppressor of cytokine signalling 1; DNMT3B, DNA methyltransferase 3B; 5-Aza, 5-aza-2'-deoxycytidine; NC, negative control; UM con, unmethylated control; M con, methylated control.

sites between miR-29a and DNMT3B are indicated in Fig. 4E. In U2OS and MG-63 cells, the relative luciferase activity in the DNMT3B-WT group was significantly downregulated by transfection with miR-29a mimics as compared with cells transfected with miR-29a NC. By contrast, no significant change was observed in the DNMT3B-MUT group upon transfection with miR-29a mimics or NC (Fig. 4F), indicating that DNMT3B is a direct target of miR-29a. Taken together, these results suggested that miR-29a upregulated SOCS1 by directly targeting DNMT3B, which inhibited the methylation level of SOCS1.

miR-29a promotes apoptosis of U2OS and MG-63 cells. Apoptosis was assayed to further investigate the effects of miR-29a on U2OS and MG-63 cells. The results demonstrated that the apoptosis rate was significantly promoted when the two cell lines were transfected with miR-29a mimics or treated with 5-Aza (Fig. 5A and B). The highest apoptosis rate was observed in cells that were simultaneously transfected with miR-29a mimics and treated with 5-Aza. When transfected with miR-29a inhibitor, the cell apoptosis was slightly suppressed, although no significant difference was observed (Fig. 5C and D).

Furthermore, the levels of several apoptosis-associated proteins, including uncleaved caspase-3, cleaved caspase-3, uncleaved PARP, cleaved PARP, Bax and Bcl-2, were measured. In the two cell lines, overexpression of miR-29a significantly increased the expression levels of cleaved caspase-3, cleaved PARP and Bax, whereas it markedly decreased the levels of uncleaved caspase-3, uncleaved PARP and Bcl-2. Co-treatment with 5-Aza further promoted these effects on apoptosis-associated proteins, the ratio of cleaved/uncleaved caspase-3 and cleaved/uncleaved PARP were significantly enhanced when cells were transfected with miR-29a mimics and 5-Aza (Fig. 5E and F). 

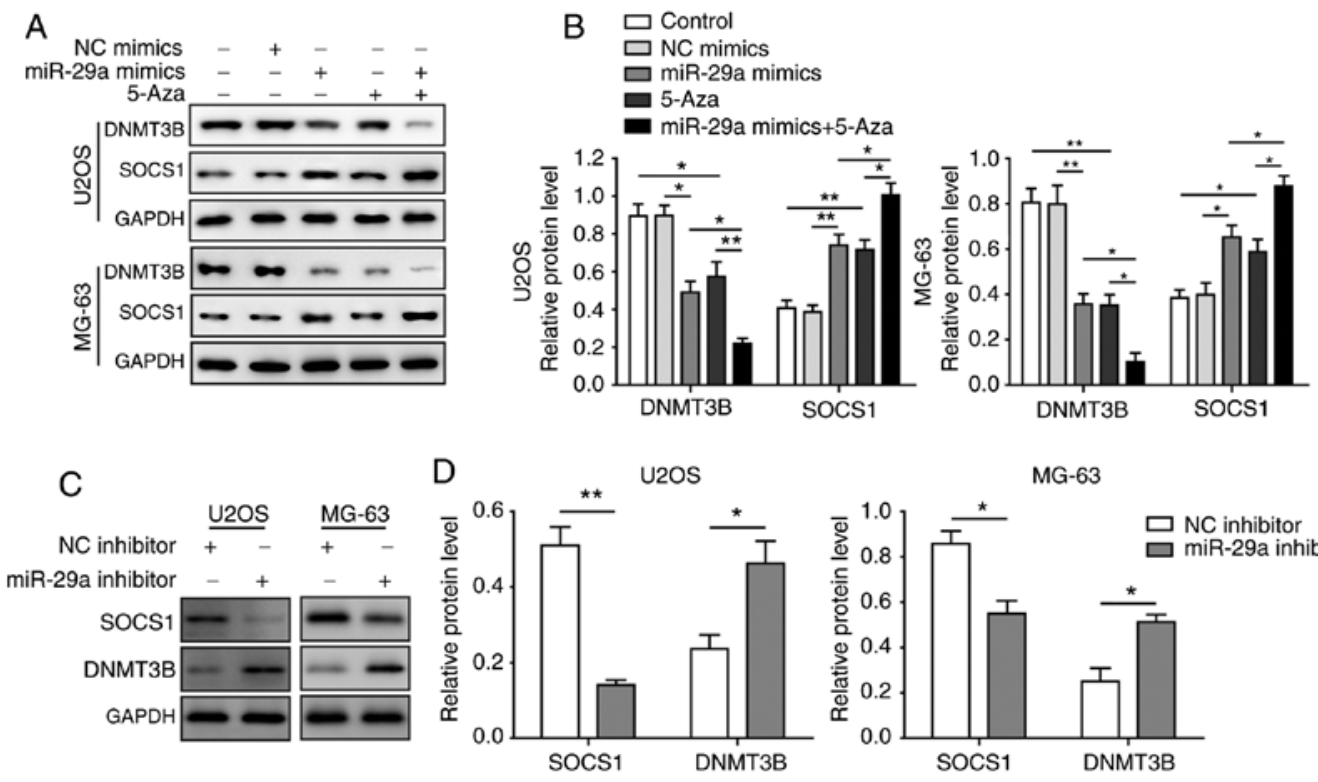

E
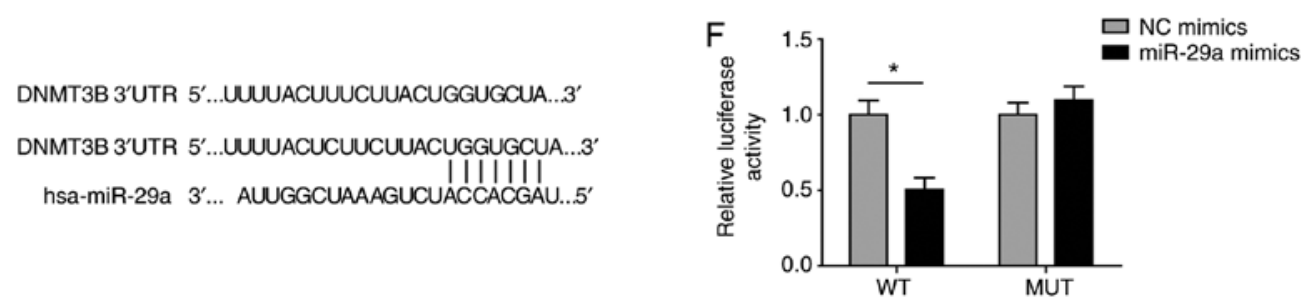

Figure 4. miR-29a downregulates the methylation of SOCS1 and upregulates SOCS1 expression by negatively targeting DNMT3B in U2OS and MG-63 cells. (A) Western blots and (B) quantified protein expression levels of DNMT3B and SOCS1 in cells transfected with miR-29a mimics and/or treated with 5-Aza. (C) Western blots and (D) quantified protein levels of DNMT3B and SOCS1 in cells transfected with miR-29a inhibitor or NC. (E) Predicted binding site of DNMT3B and miR-29a. (F) Relative luciferase activity in DNMT3B-WT and DNMT3B-MUT following transfection with miR-29a mimics or NC. Comparison between two groups was performed using unpaired Student's t-test, while comparison among three or more groups was conducted using one-way analysis of variance followed by Tukey's post hoc test. Data are expressed as the mean \pm standard deviation. " $\mathrm{P}<0.05$ and ${ }^{* * *} \mathrm{P}<0.01$. miR-29a, microRNA-29a; SOCS1, suppressor of cytokine signalling 1; DNMT3B, DNA methyltransferase 3B; 5-Aza, 5-aza-2'-deoxycytidine; NC, negative control; PARP, poly(ADP-ribose) polymerase.

miR-29a inhibits cell invasion, migration and EMT process of $U 2 O S$ and $M G-63$ cells. The effects of miR-29a on the cell invasion, migration and EMT process were subsequently investigated. The invasion and migration abilities were significantly inhibited by transfection with miR-29a mimics or treatment with 5-Aza, while the inhibitory effects were further enhanced in cells treated with the combination of miR-29a mimics and 5-Aza (Fig. 6A-D). However, when transfected with the miR-29a inhibitor, the invasion and migration abilities of the cells were evidently promoted (Fig. 6E-H). All these results indicated that miR-29a was able to promote apoptosis, as well as inhibit cell invasion and migration, in U2OS and MG-63 cells.

Furthermore, as shown in Fig. 7A and B, the levels of fibronectin, vimentin, N-cadherin, MMP-2 and MMP-9 were significantly inhibited in U2OS and MG-63 cells by overexpression of miR-29a or treatment with 5-Aza, as compared with the control group. However, the level of E-cadherin was markedly increased in the miR-29a mimics or 5-Aza groups. Combination treatment with miR-29a mimics and 5-Aza further promoted the aforementioned effects, suggesting that miR-29a inhibited the EMT process of U2OS and MG-63 cells.

miR-29a inhibits $N F-\kappa B$ signalling pathway to suppress $O S$ tumourigenesis. Considering that SOCS1 can affect NF- $\mathrm{NB}$ signalling and thus influence the invasion and migration of cancer cells, the current study subsequently investigated the effects of miR-29a and 5-Aza on NF- $\mathrm{NB}$ signalling. The results revealed that the expression of cytoplasmic p65 was significantly elevated and that of nuclear p65 was significantly decreased when cells were transfected with miR-29a mimics or treated with 5-Aza, indicating that the nuclear translocation of p65 was significantly inhibited by miR-29a and 5-Aza. In addition, the expression of IкB- $\alpha$ was significantly enhanced, while $\mathrm{p}-\mathrm{I} \kappa \mathrm{B}-\alpha$ expression and the ratio of $\mathrm{p}-\mathrm{I} \kappa \mathrm{B}-\alpha / \mathrm{I} \kappa \mathrm{B}-\alpha$ were significantly decreased when cells were transfected with miR-29a mimics or treated with 5-Aza. All these effects were most significant under the combination of miR-29a mimics and 5-Aza (Fig. 8A and B). However, when cells were transfected with miR-29a inhibitor, the opposite results were observed. Upon the suppression of miR-29a, the expression of cytoplasmic p65 was significantly decreased, while nuclear p65 was significantly elevated. Furthermore, the expression of $\mathrm{I} \kappa \mathrm{B}-\alpha$ was significantly decreased, while the expression of $\mathrm{p}-\mathrm{I} \kappa \mathrm{B}-\alpha$ and the ratio of p-IкB- $\alpha /$ I $\kappa$ B- $\alpha$ were markedly enhanced (Fig. 9A and B). These results collectively suggested that miR-29a inhibited the activation of NF- $\mathrm{BB}$ signalling, which modulated OS tumourigenesis.

miR-29a suppresses the invasion and migration of OS cells through the SOCS1/NF- $\kappa B$ signalling pathway. Finally, cells were co-transfected with siSOCS1 and miR-29a mimics to examine the effects on cell apoptosis, invasion and migration. The results revealed that, when co-transfected with siSOCS1 
A
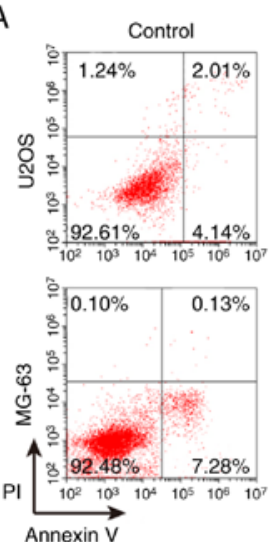

B
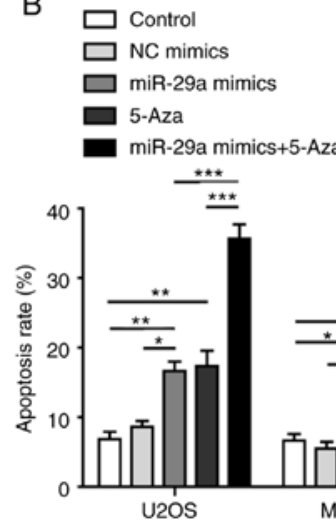

D

$\square$ NC inhibitor $\square$ miR-29a inhibitor

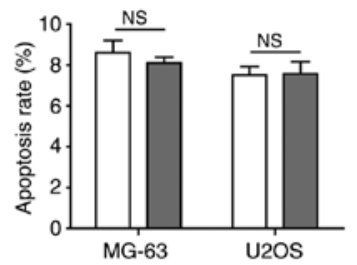

$\mathrm{F}$

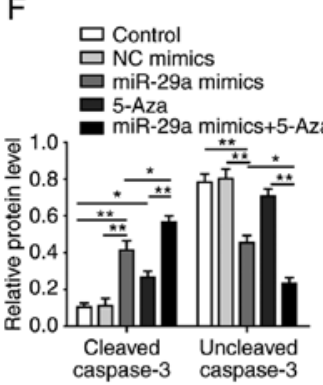

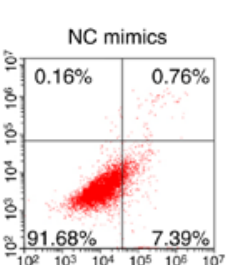
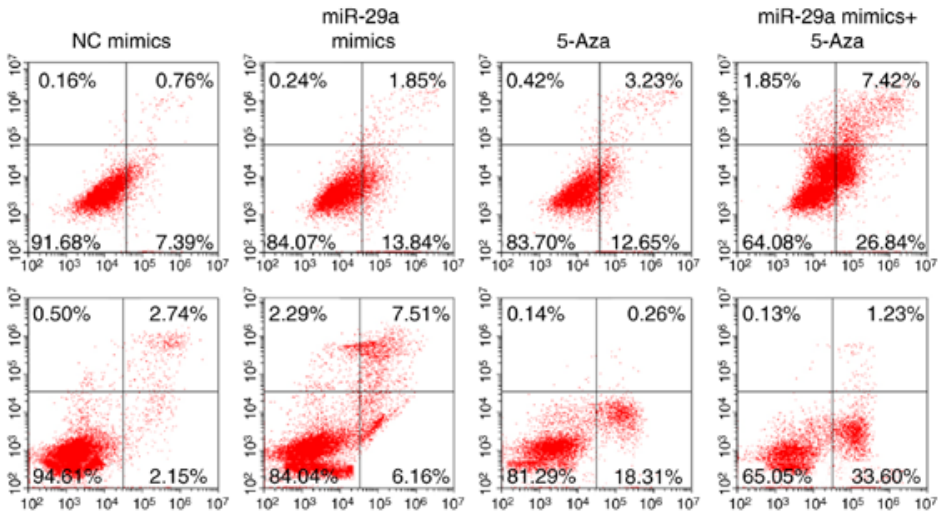

C

NC inhibitor miR-29a inhibitor
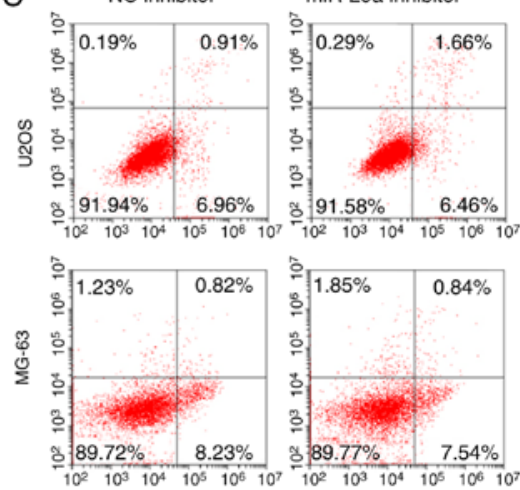

MG-63
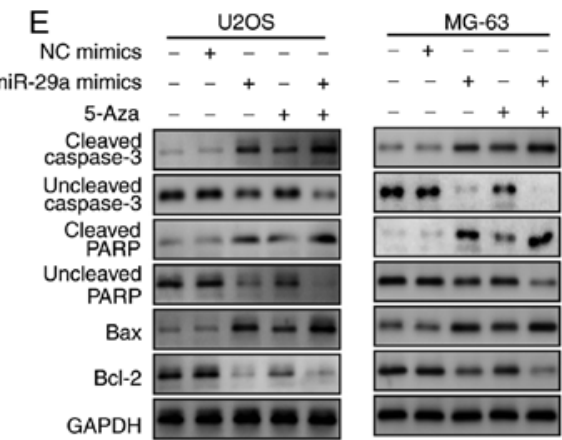

U2OS
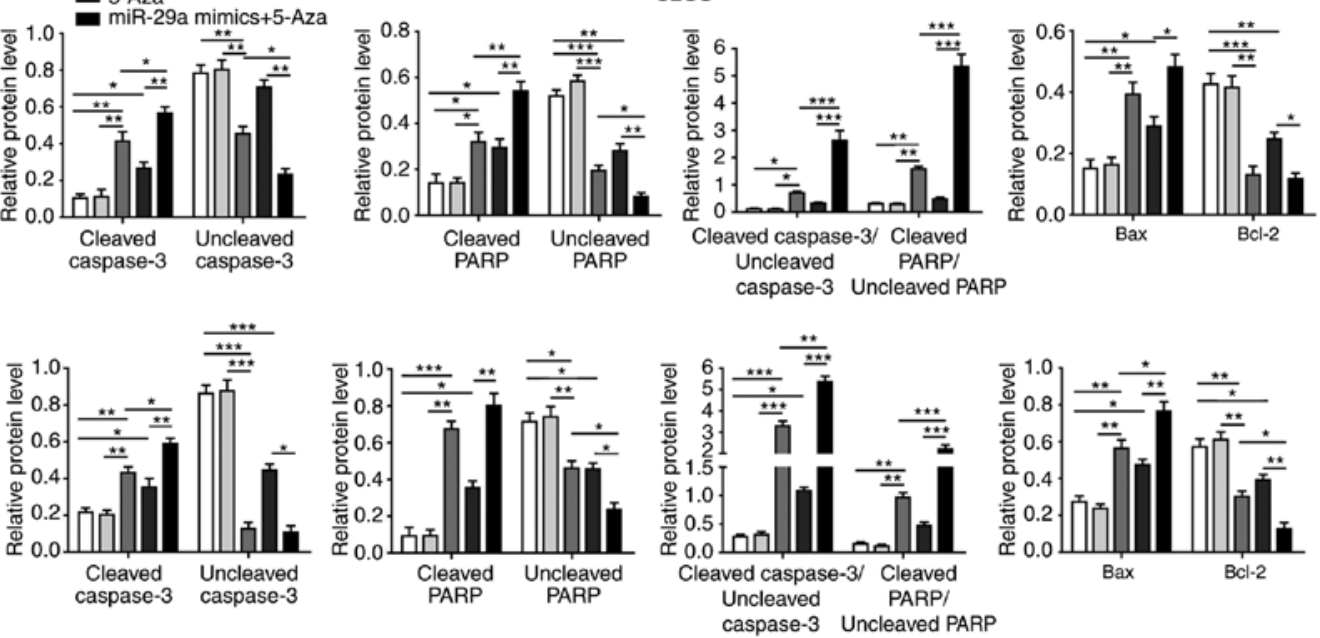

Figure 5. miR-29a promotes apoptosis of U2OS and MG-63 cells. (A) Flow cytometry results and (B) quantified apoptosis rate of cells transfected with miR-29a mimics and/or treated with 5-Aza. (C) Flow cytometry results and (D) quantified apoptosis rate of cells transfected with miR-29a inhibitor or NC. (E) Western blots and (F) quantified protein levels of cleaved caspase-3, uncleaved caspase-3, cleaved PARP, uncleaved PARP, Bax and Bcl-2. Data are expressed as the mean \pm standard deviation. Comparison between two groups was performed using unpaired Student's t-test, while comparison among three or more groups was conducted using one-way analysis of variance followed by Tukey's post hoc test. ${ }^{*} \mathrm{P}<0.05,{ }^{* *} \mathrm{P}<0.01$ and ${ }^{* * *} \mathrm{P}<0.001$. miR-29a, microRNA-29a; NC, negative control; PARP, poly(ADP-ribose) polymerase; Bcl-2, B-cell lymphoma-2; Bax, Bcl-2-associated X protein; N.S., non-significant. 

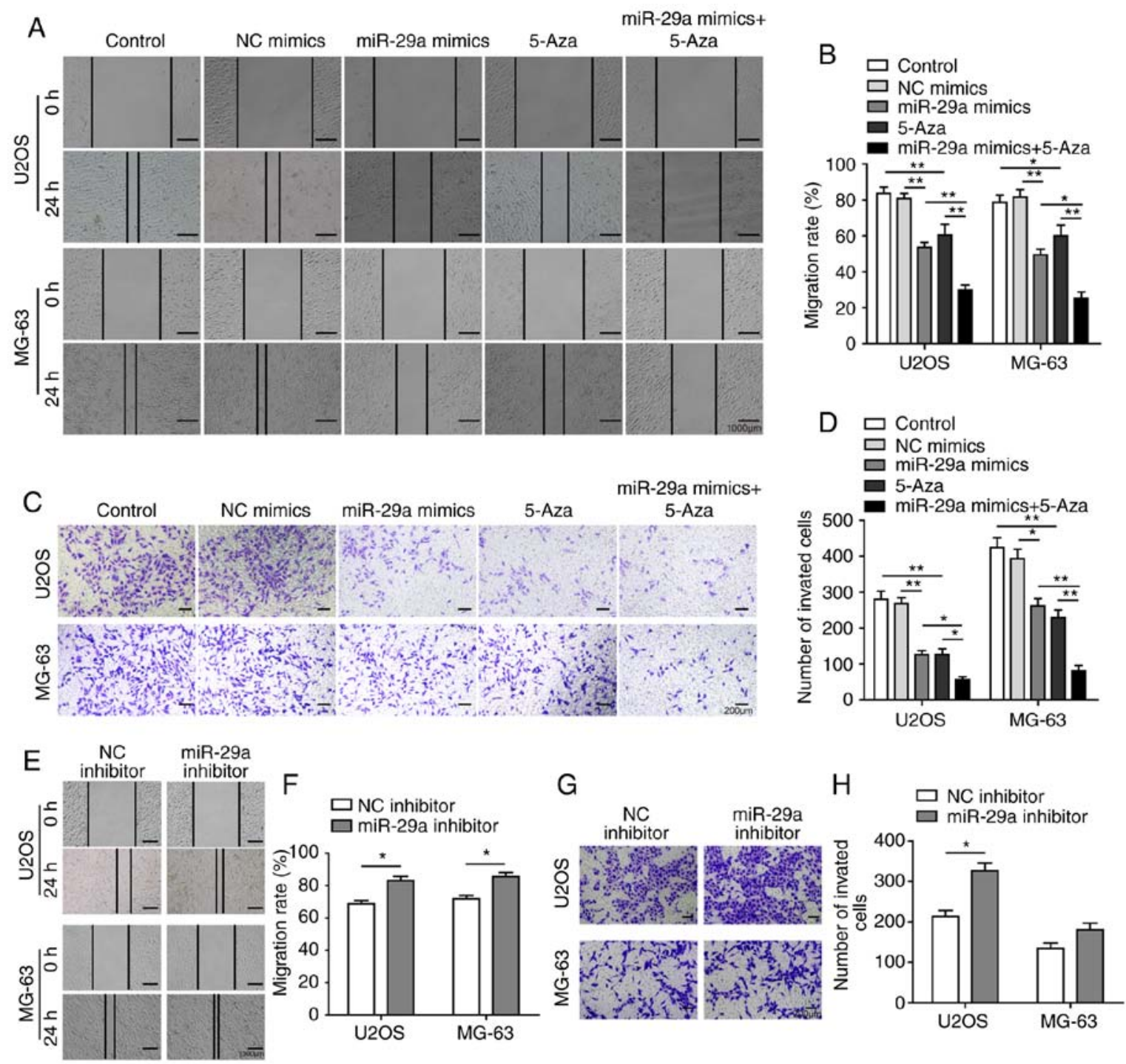

Figure 6. miR-29a inhibits invasion and migration of U2OS and MG-63 cells. (A) Representative wound healing images (magnification, $\mathrm{x} 40$ ) and (B) quantified migration rate of cells transfected with miR-29a mimics and/or treated with 5-Aza. (C) Representative Transwell invasion assay images (magnification, x100) and (D) number of invaded cells transfected with miR-29a mimics and/or treated with 5-Aza. (E) Representative wound healing images (magnification, x40) and (F) quantified migration rate of cells transfected with miR-29a inhibitor or NC. (G) Representative Transwell invasion assay images (magnification, x100) and $(\mathrm{H})$ number of invaded cells transfected with miR-29a inhibitor and/or NC. Comparison between two groups was performed using unpaired Student's $\mathrm{t}$-test, while comparison among three or more groups was conducted using one-way analysis of variance followed by Tukey's post hoc test. "P $<0.05$ and ${ }^{* * *} \mathrm{P}<0.01$. miR-29a, microRNA-29a; NC, negative control; MMP, matrix metalloproteinase.

and miR-29a mimics, the miR-29a-induced increase in SOCS1 expression was significantly decreased by siSOCS1 (Fig. 10A-C). The cell apoptosis rate exhibited no significant difference in cells transfected with siSOCS1 compared with the control group. However, the apoptosis rate was increased by miR-29a mimics, whereas this was markedly decreased when co-transfected with siSOCS1 and miR-29a mimics (Fig. 10D and E), suggesting that transfection with siSOCS1 reversed the effects of miR-29a treatment. Furthermore, the detection of apoptosis-associated proteins demonstrated that miR-29a mimics significantly enhanced the expression levels of cleaved caspase-3, cleaved PARP and Bax, while they markedly decreased the levels of uncleaved caspase-3, uncleaved PARP and Bcl-2; however, co-transfection with siSOCS1 reversed these effects (Fig. 10F and G). Additionally, the miR-29a mimics-induced ratio of cleaved/uncleaved caspase-3 and cleaved/uncleaved PARP were markedly reversed by the knockdown of SOCS1 as compared with the miR-29a mimics alone (Fig. 10F and G). Similarly, the inhibition of cell invasion and migration abilities of miR-29a mimics were reversed by co-transfection with siSOCS1 (Fig. 11A-D). All these results further confirmed that miR-29a suppressed the invasion and migration of OS cells through the SOCS1/NF- $\mathrm{B}$ signalling pathway.

\section{Discussion}

Despite developments in therapeutic methods and diagnosis, the survival rate of OS patients remains poor due to the high aggressiveness and metastasis properties of this tumour. miR-29a, which is considered to be a tumour suppressor, has been reported to be abnormally expressed in OS (12). However, to date, no study has focussed on the roles of the miR-29a/DNMT3B/SOCS1 axis in the invasion and migration of OS. In the present study, it was demonstrated for the first 


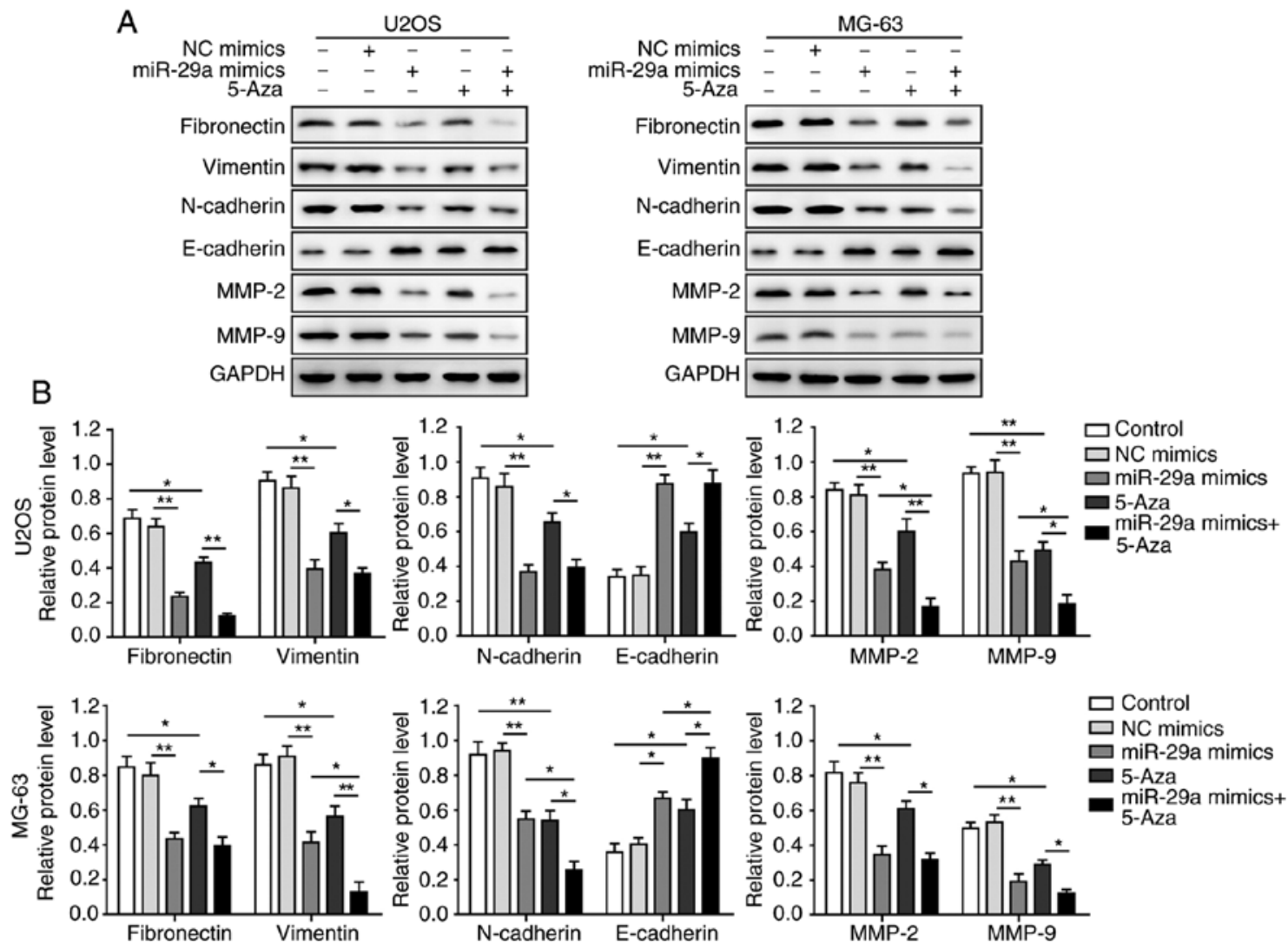

Figure 7. miR-29a inhibits epithelial-mesenchymal transition process of U2OS and MG-63 cells. (A) Western blots and (B) quantified protein expression levels of fibronectin, vimentin, N-cadherin, MMP-2, MMP-9 and E-cadherin. Data are expressed as the mean \pm standard deviation. Comparison among three or more groups was conducted using one-way analysis of variance followed by Tukey's post hoc test. "P<0.05 and ${ }^{* *} \mathrm{P}<0.01$. miR-29a, microRNA-29a; NC, negative control; MMP, matrix metalloproteinase.

time that miR-29a promoted apoptosis, and inhibited invasion, migration and EMT in OS cells by directly targeting DNMT3B and then suppressing the methylation level of SOCS1. In addition, these effects were found to be associated with inhibition of the NF- $\kappa B$ signalling pathway.

The clinical significance of miR-29a, DNMT3B and SOCS1 in cancer has been reported in several studies. It has been reported that miR-29a was significantly upregulated in tissue samples of colorectal cancer patients and may be used as a promising diagnostic biomarker (31). Zhang et al (32) revealed that miR-29a and miR-29b were downregulated in OS tissues. Furthermore, Liu et al (15) reported that overexpression of DNMT3B was correlated to the downregulation of miR-29a in juvenile myelomonocytic leukaemia patients. Lv et al (33) further demonstrated that SOCS1 expression was significantly lower in breast cancer tissues, and was correlated with lymph node metastasis and clinical staging. In the present study, it was also observed that miR-29a and SOCS1 were downregulated, and DNMT3B was upregulated in OS tissues and cells. In addition, miR-29a and SOCS1 expression levels were associated with advanced clinical stage and distant metastasis.

The role of miR-29a as a tumour suppressor, and the effects of SOCS1 and DNMT3B on tumour development have been demonstrated in several previous studies. For instance, it has been reported that miR-29a suppresses cell proliferation and migration by downregulating IGF1R in hepatocellular carcinoma (34). In addition, the downregulation of SOCS1 promotes cell growth and tumourigenesis in gastric cancer (35). Another study indicated that mahanine induced the demethylation of the RASSF1A promoter in prostate cancer cells by downregulating DNMT1 and DNMT3B (36).

Several related studies have focussed on the association of miR-29a with SOCS1 or DNMT3B. Chen et al (37) reported that miR-29a was able to promote metastasis of hepatocellular carcinoma through the ten-eleven translocation (TET)-SOCS1-MMP-9 signalling axis. DNMT3B was also revealed to be a target of miR-29a in neuroblastoma (38). Recently, Fu et al (39) demonstrated that the upregulation of DNMT3A/3B could enhance the methylation level of SOCS1-CpG islands. However, no study has reported whether miR-29a influences the expression of DNMT3B or affects the methylation of SOCS1 in OS cells. In the present study, using a dual-luciferase reporter assay, it was observed that miR-29a directly targets DNMT3B. It was also demonstrated for the first time that miR-29a promoted the apoptosis, and inhibited the invasion, migration and EMT of OS cells by directly targeting DNMT3B and then downregulating the methylation level of SOCS1.

The regulatory effect of SOCS1 on the EMT and NF- $\mathrm{KB}$ signalling is well demonstrated. SOCS1 regulates the EMT and metastasis of prostate cancer (20). In the present study, it was demonstrated that the inhibition of NF- $\kappa \mathrm{B}$ signalling was also involved in the aforementioned process. Gebeshuber et al (40) reported that miR-29a suppressed EMT and metastasis in lung cancer. The study by Kogure et al (41) further demonstrated that miR-29a was associated with epigenetic regulation of 

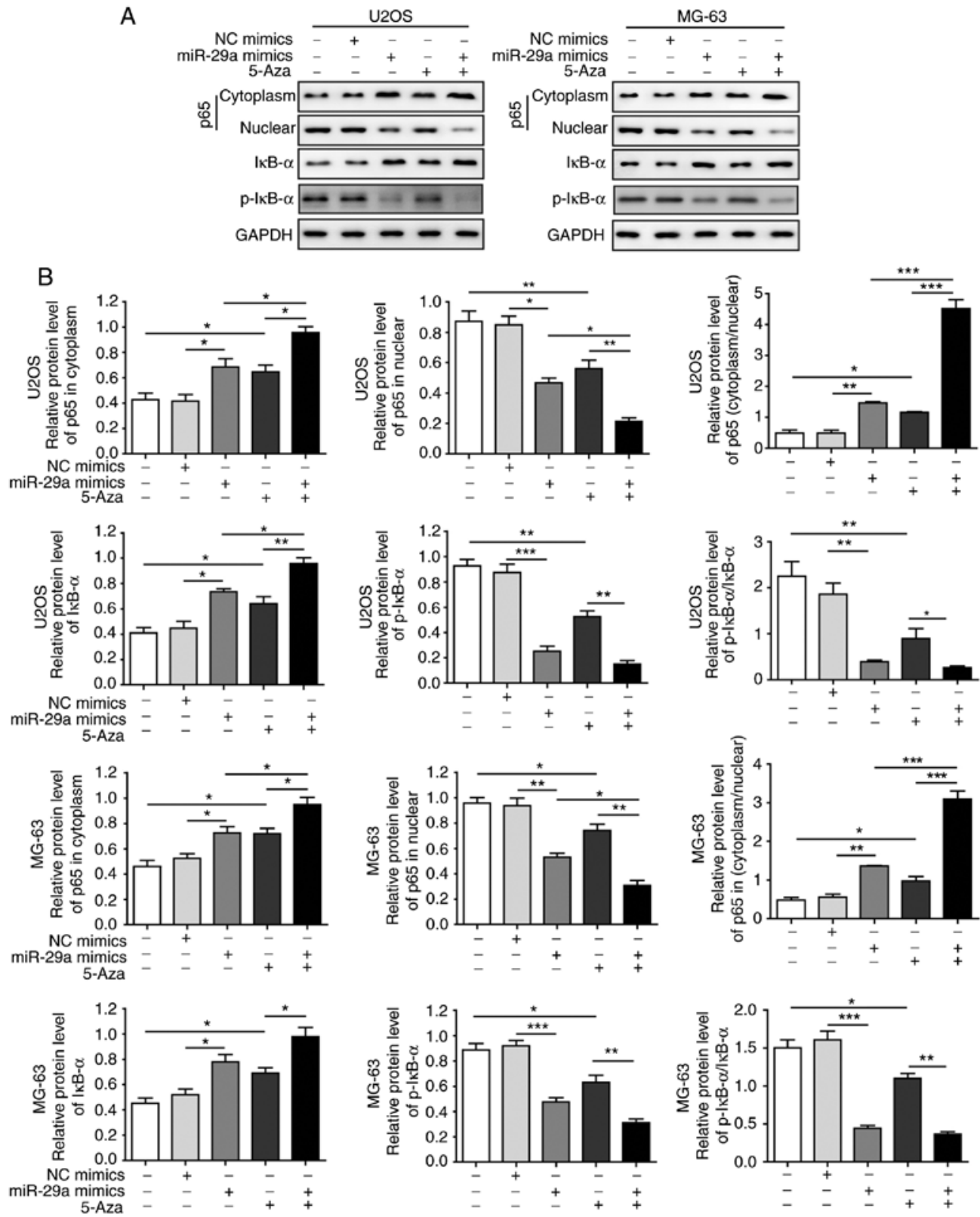

Figure 8. miR-29a inhibits the nuclear factor- $\kappa \mathrm{B}$ signalling pathway to suppress osteosarcoma tumourigenesis. (A) Western blots and (B) quantified protein

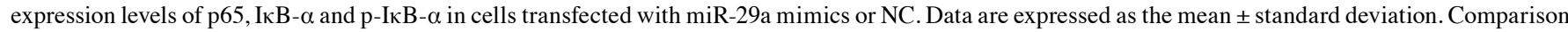
among three or more groups was conducted using one-way analysis of variance followed by Tukey's post hoc test. ${ }^{*} \mathrm{P}<0.05,{ }^{* *} \mathrm{P}<0.01$ and ${ }^{* * *} \mathrm{P}<0.001$. miR-29a, microRNA-29a; NC, negative control.

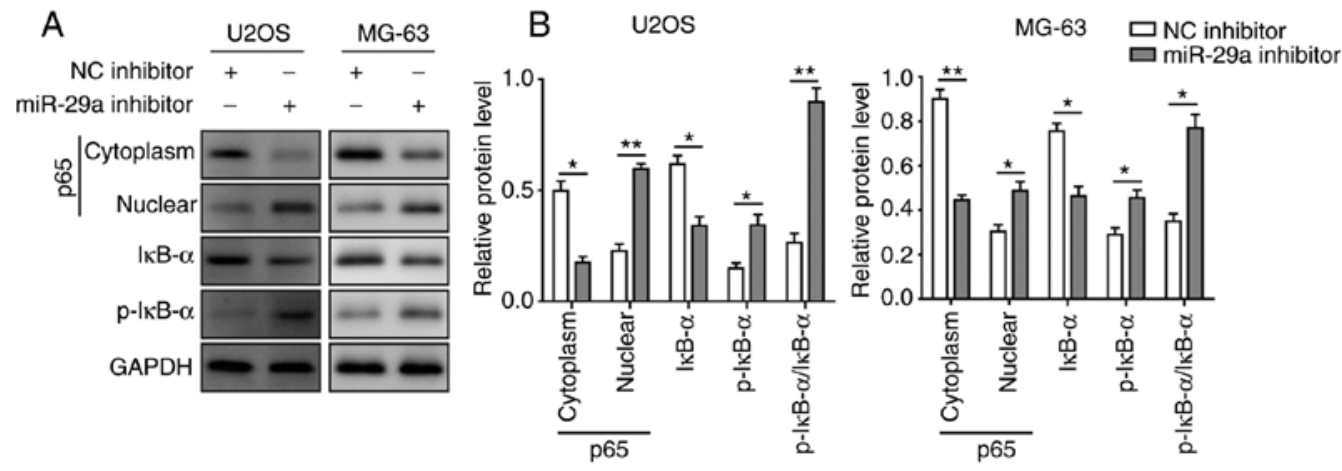

Figure 9. Inhibition of miR-29a activates the nuclear factor- $\kappa \mathrm{B}$ signalling pathway in U2OS and MG-63 cells. (A) Western blots and (B) quantified protein

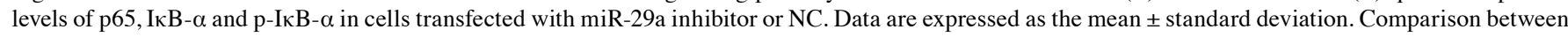
two groups was performed using unpaired Student's t-test, while comparison among three or more groups was conducted using one-way analysis of variance followed by Tukey's post hoc test. ${ }^{*} \mathrm{P}<0.05$ and ${ }^{* *} \mathrm{P}<0.01$. miR-29a, microRNA-29a; NC, negative control. 


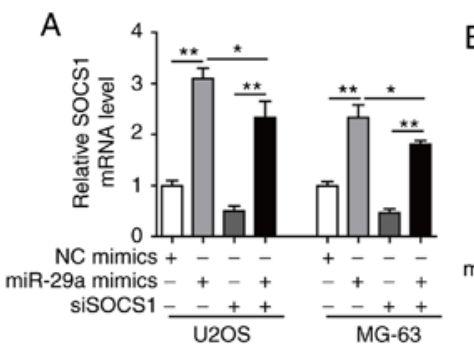

B

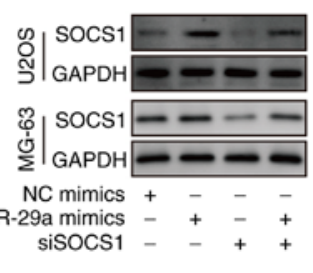

C

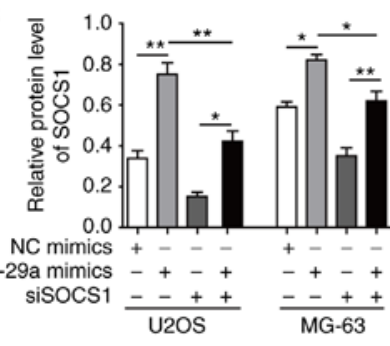

D
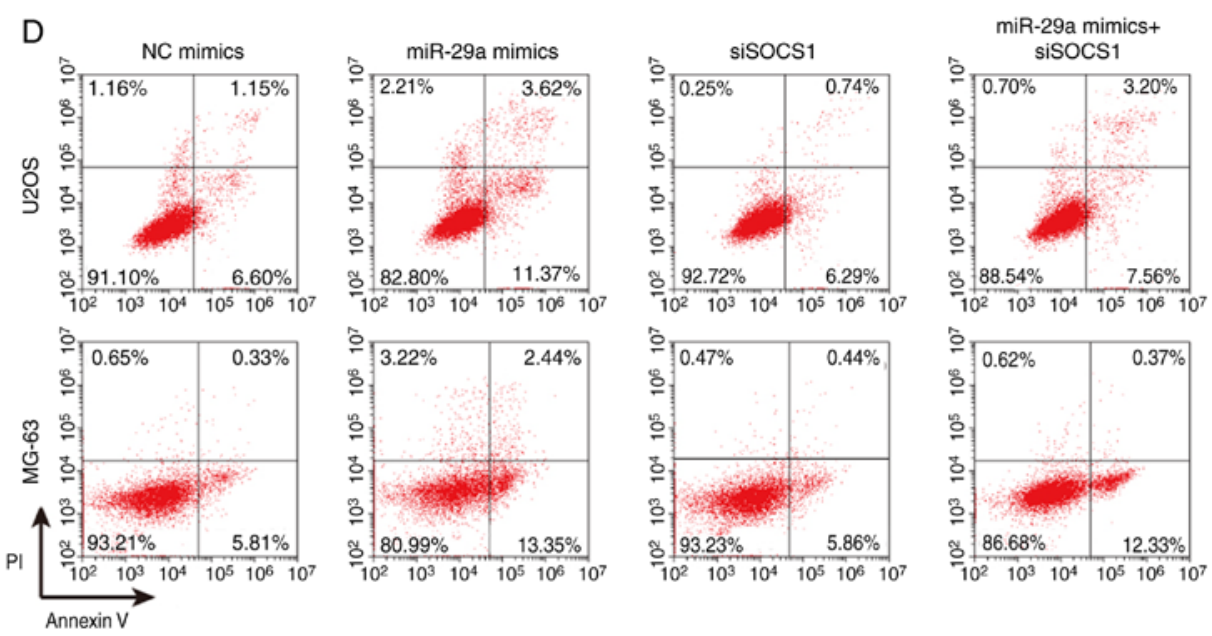
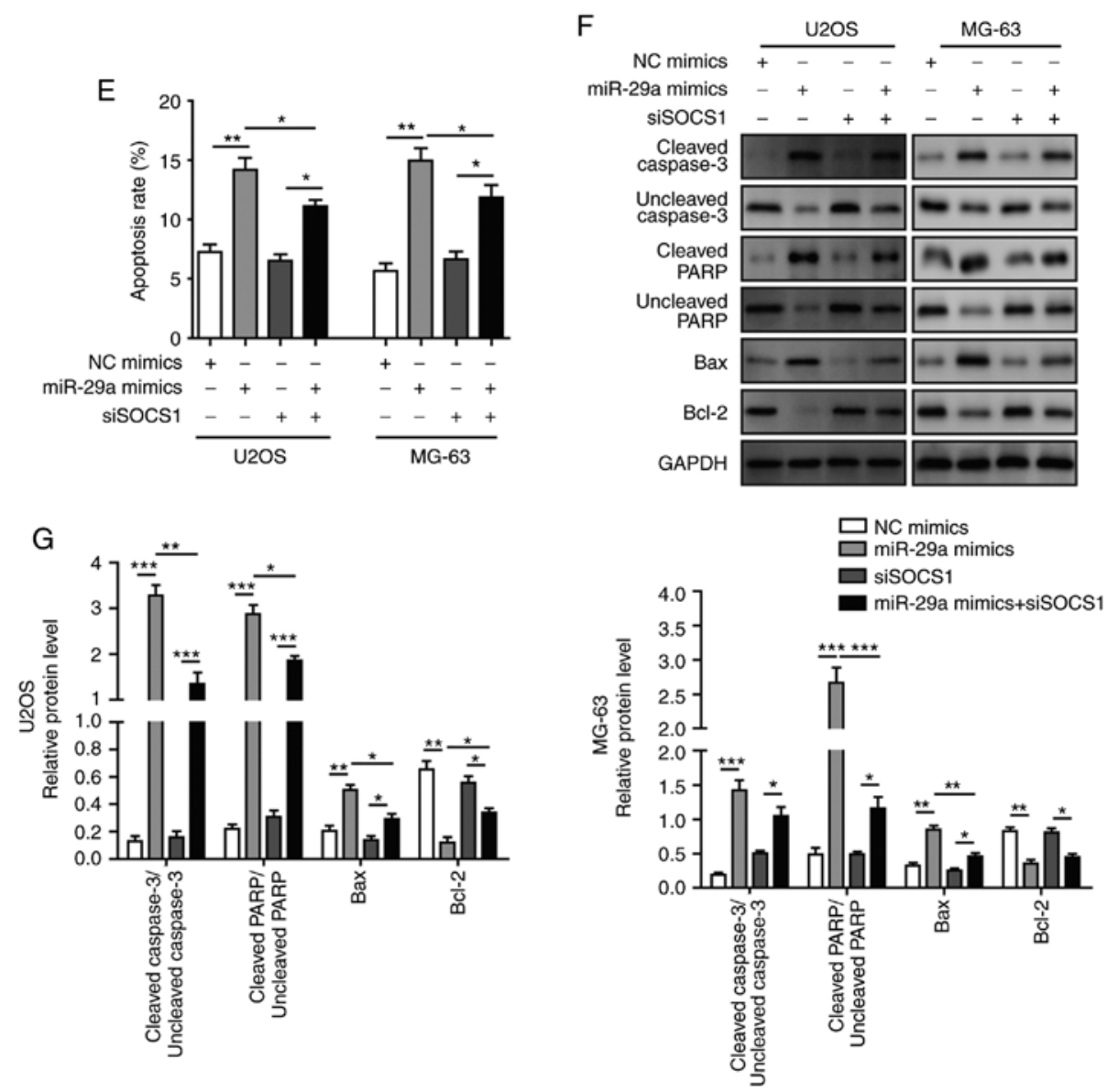

Figure 10. miR-29a promotes cell apoptosis of U2OS and MG-63 osteosarcoma cells through the SOCS1/NF- $\mathrm{KB}$ signalling pathway. (A) mRNA expression of SOCS1 in cells transfected with miR-29a mimics and/or siSOCS1, assessed by reverse transcription-quantitative polymerase chain reaction. (B) Western blots and (C) quantified protein level of SOCS1 in cells transfected with miR-29a mimics and/or siSOCS1. (D) Flow cytometry results and (E) quantified apoptosis rate of cells transfected with miR-29a mimics and/or siSOCS1. (F) Western blots and (G) quantified protein levels of cleaved caspase-3, uncleaved caspase-3, cleaved PARP, uncleaved PARP, Bax and Bcl-2 in cells transfected with miR-29a mimics and/or siSOCS1. Data are expressed as the mean \pm standard deviation. Comparison among three or more groups was conducted using one-way analysis of variance followed by Tukey's post hoc test. ${ }^{*} \mathrm{P}<0.05,{ }^{* *} \mathrm{P}<0.01$ and ${ }^{* * *} \mathrm{P}<0.001$. miR-29a, microRNA-29a; SOCS1, suppressor of cytokine signalling 1; siSOCS1, SOCS1 siRNA; NC, negative control; PARP, poly (ADP-ribose) polymerase; Bcl-2, B-cell lymphoma-2; Bax, Bcl-2-associated X protein. 


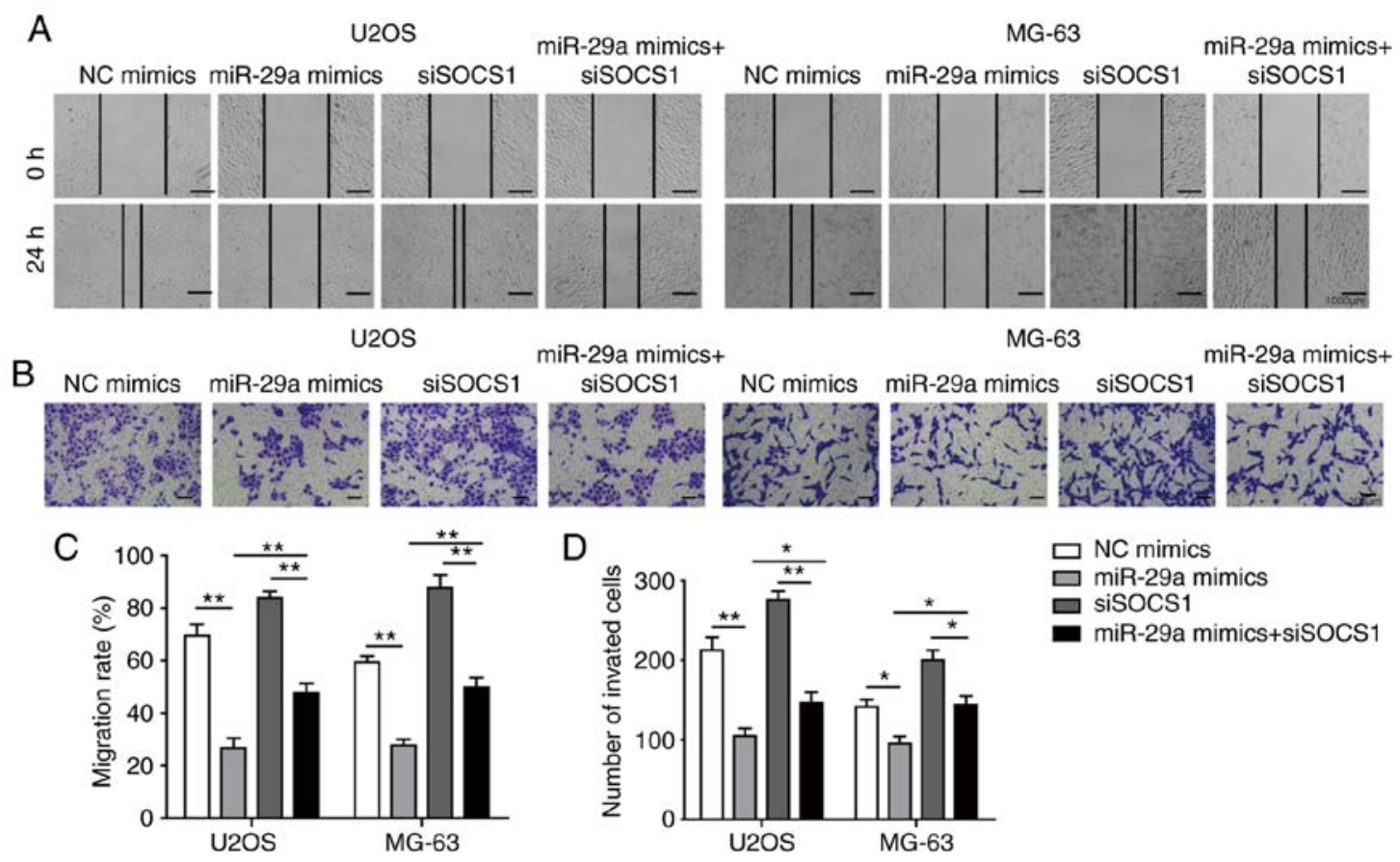

Figure 11. miR-29a suppresses the invasion and migration of U2OS and MG-63 osteosarcoma cells through the SOCS1/NF- $\mathrm{kB}$ signalling pathway. (A) Migration of cells transfected with miR-29a mimics and/or siSOCS1, examined by wound healing assay (magnification, $\mathrm{x} 40$ ). (B) Invasion of cells transfected with miR-29a mimics and/or siSOCS1, as measured by the Transwell assay (magnification, x100). (C) Quantified cell migration rate and (D) number of invaded cells. Data are expressed as the mean \pm standard deviation. Comparison among three or more groups was conducted using one-way analysis of variance followed by Tukey's post hoc test. "P<0.05 and "** $\mathrm{P}<0.01$. miR-29a, microRNA-29a; SOCS1, suppressor of cytokine signalling 1; siSOCS1, SOCS1 siRNA; NC, negative control.

transforming growth factor $\beta$-induced EMT in hepatocellular carcinoma. Furthermore, miR-29a was reported to regulate the lipopolysaccharide-induced inflammatory responses through the Akt1/NF- $\kappa \mathrm{B}$ pathway (42). In the current research, the results revealed that $\mathrm{miR}-29 \mathrm{a}$ inhibited the $\mathrm{NF}-\kappa \mathrm{B}$ signalling pathway in OS, and the inhibition effect of miR-29a on cell invasion, migration and EMT in OS cells was reversed by inhibition of SOCS1, indicating that the effects of this miRNA may be through the inhibition of SOCS1/NF- $\kappa \mathrm{B}$ signalling.

In conclusion, this study investigated the roles of the miR-29a/DNMT3B/SOCS1 axis on the invasion and migration of OS cells. The results revealed that miR-29a promoted the apoptosis and inhibited the metastasis of OS cells via inhibition of the SOCS1/NF- $\mathrm{BB}$ signalling pathway by directly targeting DNMT3B. The current study may provide deeper insights into the role of the miR-29a/DNMT3B/SOCS1 axis in the development of OS, as well as provide novel therapeutic targets for OS treatment.

\section{Acknowledgements}

The authors would like to express their sincere gratitude to the reviewers for their constructive comments.

\section{Funding}

No funding was received.

\section{Availability of data and materials}

All data generated or analysed during this study are included in this published article.

\section{Authors' contributions}

JDN conceived the study. HLG, YT, XZM, DS, DY and JDN collected the data. HLG, YT and JDN analysed the data. HLG, YT, XZM, DYS, DY and JDN performed the experiments. DS and JDN provided the resources and supervised the study. HLG wrote the original draft. DYS and JDN reviewed and edited the manuscript. All authors read and approved the final manuscript.

\section{Ethics approval and consent to participate}

The study was approved by the Ethics Committee of the Second Xiangya Hospital, Central South University. Written informed consent was obtained from all patients prior to participation.

\section{Patient consent for publication}

Written informed consent for publication was obtained from all patients.

\section{Competing interests}

The authors declare that they have no competing interests.

\section{References}

1. Mirabello L, Troisi RJ and Savage SA: Osteosarcoma incidence and survival rates from 1973 to 2004: Data from the surveillance, epidemiology, and end results program. Cancer 115: 1531-1543, 2009.

2. Luetke A, Meyers PA, Lewis I and Juergens H: Osteosarcoma treatment-where do we stand? A state of the art review. Cancer Treat Rev 40: 523-532, 2014. 
3. Broadhead ML, Clark JC, Myers DE, Dass CR and Choong PF: The molecular pathogenesis of osteosarcoma: A review. Sarcoma 2011: 959248, 2011.

4. Kansara M, Teng MW, Smyth MJ and Thomas DM: Translational biology of osteosarcoma. Nat Rev Cancer 14: 722-735, 2014.

5. Di Fiore R, Drago-Ferrante R, Pentimalli F, Di Marzo D, Forte IM, D'Anneo A, Carlisi D, De Blasio A, Giuliano M, Tesoriere G, et al: MicroRNA-29b-1 impairs in vitro cell proliferation, self-renewal and chemoresistance of human osteosarcoma 3AB-OS cancer stem cells. Int J Oncol 45: 2013-2023, 2014.

6. Durfee RA, Mohammed M and Luu HH: Review of osteosarcoma and current management. Rheumatol Ther 3: 221-243, 2016.

7. Lin S and Gregory RI: MicroRNA biogenesis pathways in cancer. Nat Rev Cancer 15: 321-333, 2015.

8. Xie B,Ding Q,Han H and Wu D: miRCancer: A microRNA-cancer association database constructed by text mining on literature. Bioinformatics 29: 638-644, 2013.

9. Dinh TK, Fendler W, Chałubińska-Fendler J, Acharya SS O'Leary C, Deraska PV, D'Andrea AD, Chowdhury D and Kozono D: Circulating miR-29a and miR-150 correlate with delivered dose during thoracic radiation therapy for non-small cell lung cancer. Radiat Oncol 11: 61, 2016.

10. Pasqualini L, Bu H, Puhr M, Narisu N, Rainer J, Schlick B, Schäfer G, Angelova M, Trajanoski Z, Börno ST, et al: miR-22 and miR-29a are members of the androgen receptor cistrome modulating LAMC1 and Mcl-1 in prostate cancer. Mol Endocrinol 29: 1037-1054, 2015.

11. Zhang H, Bai M, Deng T, Liu R, Wang X, Qu Y, Duan J, Zhang L, Ning T, Ge S, et al: Cell-derived microvesicles mediate the delivery of miR-29a/c to suppress angiogenesis in gastric carcinoma. Cancer Lett 375: 331-339, 2016.

12. Sampson VB, Yoo S, Kumar A, Vetter NS and Kolb EA: MicroRNAs and potential targets in osteosarcoma: Review. Front Pediatr 3: 69, 2015.

13. He DX, Gu XT, Li YR, Jiang L, Jin J and Ma X: Methylationregulated miR-149 modulates chemoresistance by targeting GlcNAc N-deacetylase/N-sulfotransferase-1 in human breast cancer. FEBS J 281: 4718-4730, 2014.

14. Li X, Pan Q, Wan X, Mao Y, Lu W, Xie X and Cheng X: Methylation-associated Has-miR-9 deregulation in paclitaxelresistant epithelial ovarian carcinoma. BMC Cancer 15: 509, 2015

15. Liu YL, Lensing SY, Yan Y, Webster C and Emanuel PD: Overexpression Of DNMT3a and DNMT3b is related to downregulation of Mir-29a in juvenile myelomonocytic leukemia (JMML). Blood 122: 4890, 2013.

16. Babon JJ and Nicola NA: The biology and mechanism of action of suppressor of cytokine signaling 3. Growth Factors 30: 207-219, 2012

17. Beaurivage C, Champagne A, Tobelaim WS, Pomerleau V Menendez A and Saucier C: SOCS1 in cancer: An oncogene and a tumor suppressor. Cytokine 82: 87-94, 2016.

18. Zhang XH, Yang L, Liu XJ, Zhan Y, Pan YX, Wang XZ and Luo JM: Association between methylation of tumor suppressor gene SOCS1 and acute myeloid leukemia. Oncol Rep 40: 1008-1016, 2018

19. Huang FJ, Steeg P, Price J, Sawaya R and Huang S: Suppressor of cytokine signaling-1 (SOCS-1) negatively regulates angiogenesis, invasion, and brain metastasis of melanoma cells. Cancer Res 68, 2008.

20. Shao N, Ma G, Zhang J and Zhu W: miR-221-5p enhances cell proliferation and metastasis through post-transcriptional regulation of SOCS1 in human prostate cancer. BMC Urol 18: 14, 2018

21. Hashimoto M, Ayada T, Kinjyo I, Hiwatashi K, Yoshida H, Okada Y, Kobayashi T and Yoshimura A: Silencing of SOCS1 in macrophages suppresses tumor development by enhancing antitumor inflammation. Cancer Sci 100: 730-736, 2009.

22. Gaspericampani A, Pancotti F and Roncuzzi L: Abstract 4396 Caveolin-1 as an oncopromoter in solidtumors: A role mediated by STAT3 in vitro. Cancer Res 73 (8 Suppl): S4396-S4396, 2014

23. Zhang H, Zhao Z, Pang X, Yang J, Yu H, Zhang Y, Zhou H and Zhao J: Genistein protects against Ox-LDL-induced inflammation through microRNA-155/SOCS1-mediated repression of NF-kB signaling pathway in HUVECs. Inflammation 40 : $1450-1459,2017$

24. Lee YJ, Han JY, Byun J, Park HJ, Park EM, Chong YH, Cho MS and Kang JL: Inhibiting Mer receptor tyrosine kinase suppresses STAT1, SOCS1/3, and NF- $\mathrm{KB}$ activation and enhances inflammatory responses in lipopolysaccharide-induced acute lung injury. J Leukoc Biol 91: 921-932, 2012.
25. Ren D, Yang Q, Dai Y, Guo W, Du H, Song L and Peng X: Oncogenic miR-210-3p promotes prostate cancer cell EMT and bone metastasis via NF- $\mathrm{BB}$ signaling pathway. Mol Cancer 16: 117,2017

26. Liu X, Li J, Peng X, Lv B, Wang P, Zhao X and Yu B: Geraniin inhibits LPS-induced THP-1 macrophages switching to M1 phenotype via SOCS1/NF- $\mathrm{B}$ pathway. Inflammation 39 : 1421-1433, 2016.

27. Maier HJ, Schmidt-Strassburger U, Huber MA, Wiedemann EM, Beug $\mathrm{H}$ and Wirth T: NF-kappaB promotes epithelial-mesenchymal transition, migration and invasion of pancreatic carcinoma cells. Cancer Lett 295: 214-228, 2010.

28. Wu Y and Zhou BP: TNF-alpha/NF-kappaB/Snail pathway in cancer cell migration and invasion. Br J Cancer 102: 639-644, 2010.

29. Meister P, Konrad E, Lob G, Janka G, Keyl W and Stürz H: Osteosarcoma: Histological evaluation and grading. Arch Orthop Trauma Surg 94: 91-98, 1979 .

30. Livak KJ and Schmittgen TD: Analysis of relative gene expression data using real-time quantitative PCR and the 2(-Delta Delta C(T)) method. Methods 25: 402-408, 2001.

31. Brunet Vega A, Pericay C, Moya I, Ferrer A, Dotor E, Pisa A, Casalots A, Serra-Aracil X, Oliva JC, Ruiz A and Saigí E: microRNA expression profile in stage III colorectal cancer: Circulating miR-18a and miR-29a as promising biomarkers. Oncol Rep 30: 320-326, 2013

32. Zhang W, Qian JX, Yi HL, Yang ZD, Wang CF, Chen JY, Wei XZ, Fu Q and Ma H: The microRNA-29 plays a central role in osteosarcoma pathogenesis and progression. Mol Biol (Mosk) 46: 622-627, 2012.

33. Lv Y, Song G and Li P: Correlation of SOCS-1 gene with onset and prognosis of breast cancer. Oncol Lett 16: 383-387, 2018.

34. Wang X, Liu S, Cao L, Zhang T, Yue D, Wang L, Ping Y, He Q, Zhang C, Wang M, et al: miR-29a-3p suppresses cell proliferation and migration by downregulating IGF1R in hepatocellular carcinoma. Oncotarget 8: 86592-86603, 2017.

35. Qin S, Ai F, Ji WF, Rao W, Zhang HC and Yao WJ: miR-19a promotes cell growth and tumorigenesis through targeting SOCS1 in gastric cancer. Asian Pac J Cancer Prev 14: 835-840, 2013

36. Agarwal S, Amin KS, Jagadeesh S, Baishay G, Rao PG, Barua NC, Bhattacharya S and Banerjee PP: Mahanine restores RASSF1A expression by down-regulating DNMT1 and DNMT3B in prostate cancer cells. Mol Cancer 12: 99, 2013.

37. Chen Q, Dan Y, Zhang Y, Yu L, Li XD, Zhou ZJ, Zhou SL, Gao DM, Hu J, Jin C, et al: MicroRNA-29a induces loss of 5-hydroxymethylcytosine and promotes metastasis of hepatocellular carcinoma through a TET-SOCS1-MMP9 signaling axis. Cell Death Dis 8: e2906, 2017.

38. Maugeri M, Barbagallo D, Barbagallo C, Banelli B, Di Mauro S, Purrello F, Magro G, Ragusa M, Di Pietro C, Romani M and Purrello M: Altered expression of miRNAs and methylation of their promoters are correlated in neuroblastoma. Oncotarget 7: 83330-83341, 2016.

39. Fu X, Song X, Li Y, Tan D and Liu G: Hepatitis B virus X protein upregulates DNA methyltransferase $3 \mathrm{~A} / 3 \mathrm{~B}$ and enhances SOCS-1CpG island methylation. Mol Med Rep 13: 301-308, 2016.

40. Gebeshuber CA,Zatloukal K and Martinez J: miR-29a suppresses tristetraprolin, which is a regulator of epithelial polarity and metastasis. EMBO Rep 10: 400-405, 2009.

41. Kogure T, Kondo Y, Kakazu E, Ninomiya M, Kimura O and Shimosegawa T: Involvement of miRNA-29a in epigenetic regulation of transforming growth factor- $\beta$-induced epithelial-mesenchymal transition in hepatocellular carcinoma. Hepatol Res 44: 907-919, 2014.

42. Tang B, Li X, Ren Y, Wang J, Xu D, Hang Y, Zhou T, Li F and Wang L: MicroRNA-29a regulates lipopolysaccharide (LPS)-induced inflammatory responses in murine macrophages through the Akt1/ NF-кB pathway. Exp Cell Res 360: 74-80, 2017.

This work is licensed under a Creative Commons Attribution-NonCommercial-NoDerivatives 4.0 International (CC BY-NC-ND 4.0) License. 